\title{
Sodium nitrate has no detrimental effect on milk fatty acid profile and rumen bacterial population in water buffaloes
}

\author{
Fang Xie ${ }^{\dagger}$, Zhenhua Tang ${ }^{\dagger}$, Xin Liang, Chongli Wen, Mengwei Li, Yanxia Guo, Kaiping Peng and \\ Chengjian Yang* (i)
}

\begin{abstract}
This study evaluated the influence of dietary sodium nitrate on ruminal fermentation profiles, milk production and composition, microbial populations and diversity in water buffaloes. Twenty-four female water buffaloes were randomly divided into four groups and fed with $0,0.11,0.22,044 \mathrm{~g}$ sodium nitrate per $\mathrm{kg}$ body weight diets, respectively. Results showed that the concentration of acetate, propionate, butyrate and total VFA in all sodium nitrate-adapted water buffaloes were greater than the control group $(P<0.05)$. Although the milk fatty acids value at $0.11 \mathrm{~g}$ sodium nitrate $/ \mathrm{kg} / \mathrm{d}$ were slightly lower than other treatments, no significant differences were observed among different treatments $(P>0.05)$. Compared to the control group, the archaea richness (ace and chao1) and diversity (Shannon index) indices were increased by nitrate supplementation $(P<0.05)$. Compared with the control group, sodium nitrate did not affect bacterial abundance at the phylum and genus level, but the relative abundance of the methanogen genera was greatly changed. There was a tendency for Methanobrevibacter to decrease in the sodium nitrate group $(P=0.091)$. Comparisons of archaea communities by PCOA analysis showed significant separation between the control group and nitrate treatments $(P=0.025)$. It was concluded that added $0.11-0.44 \mathrm{~g}$ sodium nitrate $/ \mathrm{kg}$ of body weight increased the rumen VFA production and archaeal diversity of water buffaloes but had no detrimental effect on milk yield or composition, fatty acids profile, rumen methanogen or Butyrivibrio group population related to biohydrogenation.
\end{abstract}

Keywords: Sodium nitrate, Fatty acids profile, Methane, Rumen, Water buffalo

\section{Introduction}

It is reported that approximate $14.5 \%$ of all anthropogenic emissions were emitted by the livestock sector every year and has a significant negative effect on climate change (Gerber et al. 2013). Methane is the second-largest greenhouse gas and representing $16 \%$ of total global greenhouse gas emissions (Pachauri et al. 2014). Methane emission from enteric fermentation from ruminants is a

\footnotetext{
*Correspondence: ycj0746@sina.com

${ }^{\dagger}$ Fang Xie and Zhenhua Tang contributed equally to this work.

Key Laboratory of Buffalo Genetics, Breeding and Reproduction

Technology, Ministry of Agriculture and Guangxi, Buffalo Research

Institute, Chinese Academy of Agricultural Sciences, 24-1Yongwu Road,

Nanning 530001, People's Republic of China
}

dominant source of greenhouse gas. In the rumen, methanogenic archaea responsible for methanogenesis uses carbon dioxide and hydrogen as main substrates. Methane reduction from ruminants by different strategies has been investigated in many studies (Buddle et al. 2011; Zhou et al. 2011; Guyader et al. 2015; Klop et al. 2016). The efficiency of nitrate supplementation on methane reduction in ruminants has been confirmed both in vivo and in vitro (Nolan et al. 2010; Lee and Beauchemin 2014; Yang et al. 2016). However, the effects of inhibition of methanogenesis on animal product quality still need to be evaluated.

Buffalo's milk is the second-largest milk type of the world after cow milk (FAO 2020) and has higher fat,
Springer Open (c) The Author(s) 2022. Open Access This article is licensed under a Creative Commons Attribution 4.0 International License, which permits use, sharing, adaptation, distribution and reproduction in any medium or format, as long as you give appropriate credit to the original author(s) and the source, provide a link to the Creative Commons licence, and indicate if changes were made. The images or other third party material in this article are included in the article's Creative Commons licence, unless indicated otherwise in a credit line to the material. If material is not included in the article's Creative Commons licence and your intended use is not permitted by statutory regulation or exceeds the permitted use, you will need to obtain permission directly from the copyright holder. To view a copy of this licence, visit http://creativecommons.org/licenses/by/4.0/. 
protein, minerals and conjugated linoleic acid (CLA) contents than cow milk (Ahmad et al. 2013). In ruminant meat and milk, saturated fatty acids were produced from the biohydrogenation of unsaturated fatty acids and were harmful to human health (Givens and Shingfield 2004). Both molecular and metabolic hydrogen can be utilized by ruminal microorganisms during methanogenesis and the biohydrogenation of unsaturated fatty acids. Many researches have been undertaken to find sustainable ways of lowering methane emissions from ruminants. The effect of dietary nitrate on methane reduction appears effective and consistent in both in vitro and in vivo studies and also persistent in several long-term studies. Nitrates on dairy farms may come from the groundwater and plants which fertilized by commercial fertilizer and/ or animal wastes. It may be consumed by grazing ruminants on a daily basis. We found sodium nitrate may lead to lower butyrate production and increased CLA passage of the rumen in vitro, however, overall biohydrogenation was not affected by methanogenesis inhibition (Yang et al. 2019). The interactions between methanogenesis and biohydrogenation in vivo are still unclear. As far as we know, the consequences of methanogenesis were inhibited by sodium nitrate on biohydrogenation in water buffaloes are still unclear. Therefore, here we assess the influence of dietary sodium nitrate supplementation on ruminal fermentation profiles, milk production and composition, microbial populations and diversity in water buffaloes.

\section{Materials and methods}

This study was conducted at the research farm of Buffalo Research Institute, Chinese Academy of Agricultural Sciences. All animal procedures were performed according to the BRI-CAAS (Buffalo Research Institute, Chinese Academy of Agricultural Sciences) guidelines on animal experiments.

\section{Animals and experimental design}

Twenty-four healthy lactating Murrah water buffaloes with the initial body weight of $614 \pm 26 \mathrm{~kg}$ was used for this study. Water buffaloes were randomly allocated to four experimental treatments. There are six replicates per treatment and one water buffalo per replicate. The four diets are as follows: basal diet without sodium nitrate (control group), basal diet with 0.11 g sodium nitrate $/ \mathrm{kg}$ of body weight (low nitrate diet), basal diet with $0.22 \mathrm{~g}$ sodium nitrate/ $\mathrm{kg}$ of body weight (medium nitrate diet), basal diet with $0.44 \mathrm{~g}$ sodium nitrate $/ \mathrm{kg}$ of body weight (high nitrate diet). The purity of sodium nitrate was $99 \%$ (Yuan Feng Co., Ltd., Zhengzhou City, China). The dosages of sodium nitrate were based on the results of the previous studies with dairy cows and beef cattle (Lee and
Beauchemin 2014). Urea was used to maintain an isonitrogenous intake of treatments. The sodium nitrate and urea were mixed with diets before being fed to animals. The nutritional levels of the basal diets are presented in Table 1. All animals were housed in individual stalls for 56 days which included a 28-days adaptation period. In order to avoid the risk of intoxication, animals receiving diets containing sodium nitrate were acclimatized gradually to the adaptation period. The diets were offered twice daily at $0600 \mathrm{~h}$ and $1400 \mathrm{~h}$. Milk, ruminal contents and blood samples were collected during the following 28 days. All water buffaloes had free access to freshwater during the entire experimental period.

\section{Sample collection}

Feed intake was measured and representative feed samples were taken for further chemical analysis during the last week of the measurements period. Water buffaloes were milked twice daily (0600 and $1600 \mathrm{~h}$ ). Milk yields were recorded every day. Approximately $500 \mathrm{ml}$ of milk samples were taken from each water buffalo each week for milk composition and fatty acids analysis. Three water buffaloes from each group were randomly selected and used as rumen content donors on the last day of the experimental duration. Approximately $250 \mathrm{ml}$ ruminal

Table 1 Constituents and nutrient concentrations in basal diet for water buffaloes

\begin{tabular}{lc}
\hline Items & DM, \% \\
\hline Cassava residues & 13.00 \\
Elephant grass & 10.00 \\
Brewer's grain & 10.00 \\
Corn silage & 17.00 \\
Corn & 26.75 \\
Soybean meal & 3.00 \\
Wheat bran & 7.50 \\
Cottonseed meal & 8.50 \\
CaHPO & \\
Shell meal & 0.75 \\
NaCl & 0.50 \\
NaHCO & \\
Premix & 1.00 \\
Total & 1.50 \\
Nutrient concentration & 0.50 \\
GE MJ/kg & 100 \\
CP\% $_{\text {NDF\% }}$ & \\
ADF\% & 16.14 \\
\hline
\end{tabular}

Nutrient concentrations were measured according the methods described in reference (Zhang 2007); each kg premix contained: vitamin E 3000 IU, vitamin D 150,000 IU, vitamin A 500,000 IU, Cu 1.3 g, Fe 4.0 g, Mn 3.0 g, I 80 mg, Zn 6.0 g, Co $80 \mathrm{mg}$, Se $50 \mathrm{mg}$ 
contents were collected before morning feeding with stomach tubing. Ruminal contents were immediately transferred to the laboratory with an icebox. During the experimental period, blood samples were collected from each water buffalo via the jugular vein in heparinized collection tubes before the morning feeding. Haemoglobin and methaemoglobin content in blood samples were analyzed within $2 \mathrm{~h}$ after sampling using MetHb and $\mathrm{Hb}$ kits (Gu Duo Sheng Wu, Shanghai).

Five $\mathrm{ml}$ of ruminal contents were kept at $-20{ }^{\circ} \mathrm{C}$ for later DNA extraction and further microbial population and microbial diversity analysis. The remaining ruminal contents were squeezed through two layers of cheesecloth; One $10 \mathrm{ml}$ filtrate was acidified with $1 \mathrm{ml} 0.5 \mathrm{~mol} / \mathrm{l}$ $\mathrm{HCl}$ and kept at $-20{ }^{\circ} \mathrm{C}$ for subsequent ruminal $\mathrm{NH}_{3}-\mathrm{N}$ analysis. One $5 \mathrm{ml}$ filtrate was kept at $-20{ }^{\circ} \mathrm{C}$ for subsequent microbial crude protein analysis. Two $\mathrm{ml}$ of freshly prepared $25 \%$ meta-phosphoric acid were added to $8 \mathrm{ml}$ of filtrate, after centrifuged $(12,000 \mathrm{rpm}, 10 \mathrm{~min})$, the supernatant fluid was used for volatile fatty acids determination.

\section{Feed, milk and rumen fermentation samples composition analysis}

Ruminal $\mathrm{pH}$ was determined immediately after samples collection using a portable $\mathrm{pH}$ meter (HI 9024C, Hanna Instruments, USA). Milk composition was determined by MilkoScan F120 (FOSS, Denmark). Milk fatty acids were quantified by gas chromatography (Yang et al. 2019). VFA concentration was also measured by gas chromatography (Qin 1982). Microbial crude protein (MCP) also measured by the method of Makkar et al. (1982). $\mathrm{NH}_{3}-\mathrm{N}$ measured by the phenol-hypochlorite method (Weatherburn 1967). Microbial DNA was extracted from rumen contents by the bead-beating method (Yu and Morrison 2004). DNA concentration and purity were determined using a NanoDrop 1000 UV-vis spectrophotometer (Thermo Scientific, USA).

\section{Primers and real-time PCR of microbial population}

The PCR primers for total bacteria, methanogens, protozoa, fungi, B. proteoclasticus, B. fibrsolvens + Pseudobutyrivibrio spp., 'Atypical' Butyrivibrio and B. hungatei were as listed in Table 2 (Sylvester et al. 2004; Denman and McSweeney 2006; Denman et al. 2007; Shingfield et al. 2012). Amplification condition was as follows: initial denaturation at $95{ }^{\circ} \mathrm{C}$ for $3 \mathrm{~min}$, followed by 40 cycles of denaturation at $95{ }^{\circ} \mathrm{C}$ for $15 \mathrm{~s}$, annealing at the respective annealing temperature for $60 \mathrm{~s}$, except for the B. proteoclasticus and the $B$. hungatei primer sets, where the following protocol was used: initial denaturation at $95{ }^{\circ} \mathrm{C}$ for $3 \mathrm{~min}$, then 40 cycles at $95^{\circ} \mathrm{C}$ for $15 \mathrm{~s}$, annealing at the respective annealing temperature for $30 \mathrm{~s}$, and extension at $72{ }^{\circ} \mathrm{C}$ for $30 \mathrm{~s}$ (Shingfield et al. 2012). PCR products concentration were measured with a NanoDrop 1000 UV-vis spectrophotometer (Thermo Scientific, USA). For each standard, copy number concentration was calculated based on the length of the PCR product and the mass concentration (Yu et al. 2005). The real-time PCR was carried out using a Roche-480 II system with fluorescence detection of SYBR green dye. The target DNA was quantified using serial ten-fold dilutions from $10^{-1}$

Table 2 PCR primers for real time PCR

\begin{tabular}{|c|c|c|c|}
\hline Target species & Forward/reverse & Primer sequences $\left(5^{\prime}->3^{\prime}\right)$ & References \\
\hline \multirow[t]{2}{*}{ Total bacteria } & $\mathrm{F}$ & CGGCAACGAGCGCAACCC & \multirow[t]{2}{*}{ Denman and McSweeney 2006} \\
\hline & R & CCATTGTAGCACGTGTGTAGCC & \\
\hline \multirow[t]{2}{*}{ Methanogens } & $\mathrm{F}$ & TTCGGTGGATCDCARAGRGC & \multirow[t]{2}{*}{ Denman et al. 2007} \\
\hline & $\mathrm{R}$ & GBARGTCGWAWCCGTAGAATCC & \\
\hline \multirow[t]{2}{*}{ Protozoal } & $\mathrm{F}$ & GCTTTCGWTGGATGTGTATT & \multirow[t]{2}{*}{ Sylvester et al. 2004} \\
\hline & $\mathrm{R}$ & CTTGCCCTCYAATCGTWCT & \\
\hline \multirow[t]{2}{*}{ Fungi } & $\mathrm{F}$ & GAGGAAGTAAAAGTCGTAACAAGGTTTC & \multirow[t]{2}{*}{ Denman and McSweeney 2006} \\
\hline & $\mathrm{R}$ & CAAATTCACAAAGGGTAGGATGATT & \\
\hline \multirow[t]{3}{*}{ B. proteoclasticus } & CprF & TCCGGTGGTATGAGATGGGC & \multirow[t]{3}{*}{ Shingfield et al. 2012} \\
\hline & CprR & GTCGCTGCATCAGAGTTTCCT & \\
\hline & CprP & CCGCTTGGCCGTCCGACCTCTCAGTCCGAGCGG & \\
\hline \multirow{2}{*}{$\begin{array}{l}\text { B. fibrisolvens + Pseudobutyrivi- } \\
\text { brio spp. }\end{array}$} & BfiF & GCCTCAGCGTCAGTAATCG & \multirow[t]{2}{*}{ Shingfield et al. 2012} \\
\hline & BfiR & GGAGCGTAGGCGGTTTTAC & \\
\hline \multirow[t]{2}{*}{ 'Atypical' Butyrivibrio } & $A t b F$ & GACGGTGTATCAAGTCTGAAGTG & \multirow[t]{2}{*}{ Shingfield et al. 2012} \\
\hline & AtbR & GCCGGCACTGAAAGACTATGTC & \\
\hline \multirow[t]{2}{*}{ B. hungatei } & BhuF & AGGGTAATGCCTGTAGCTC & \multirow[t]{2}{*}{ Shingfield et al. 2012} \\
\hline & BhuR & TCACCCTCGCGGGAT & \\
\hline
\end{tabular}


to $10^{-10}$ DNA copies of the previously quantified DNA standards.

\section{S RNA gene amplification}

The variable region V3-V4 of the bacterial 16S rRNA gene was amplified with primers 338F (5'-ACTCCT ACGGGAGGCAGCAG-3') and 806R (5'-GGACTA CHVGGGTWTCTAAT- $3^{\prime}$ ). The variable V4 region of the archaeal 16S rRNA gene was amplified using the primers Arch349F (5'-GYGCASCAGKCGMGAAW-3') and Arch806R (5'-GGACTACVSGGGTATCTAAT- $3^{\prime}$ ) (Takai and Horikoshi 2000). PCR amplification of $16 \mathrm{~S}$ rRNA genes was performed as follows: initial denaturation at $95{ }^{\circ} \mathrm{C}$ for $3 \mathrm{~min}$, followed by 27 cycles of denaturing at $95{ }^{\circ} \mathrm{C}$ for $30 \mathrm{~s}$, annealing at $55{ }^{\circ} \mathrm{C}$ for $30 \mathrm{~s}$ and extension at $72{ }^{\circ} \mathrm{C}$ for $45 \mathrm{~s}$, and single extension at $72{ }^{\circ} \mathrm{C}$ for $10 \mathrm{~min}$. PCR reactions were performed in triplicate. The PCR product was extracted from $2 \%$ agarose gel and purified using the AxyPrep DNA Gel Extraction Kit (Axygen Biosciences, USA) according to manufacturer's instructions and quantified using a Quantus ${ }^{\mathrm{TM}}$ Fluorometer (Promega, USA).

\section{Illumina MiSeq sequencing}

Purified amplicons were pooled in equimolar proportions and paired-end sequenced $(2 \times 300)$ on an Illumina MiSeq platform (Illumina, USA) according to the standard protocols by Majorbio Bio-Pharm Technology Co. Ltd. (Shanghai, China).

\section{Processing of sequencing data}

The raw $16 \mathrm{~S}$ rRNA gene sequencing reads were demultiplexed, quality-filtered by Trimmomatic and merged by FLASH. Operational taxonomic units (OTUs) with 97\% similarity cutoff were clustered using UPARSE (version 7.1, http://drive5.com/uparse/), and chimeric sequences were identified and removed. The taxonomy of each OTU representative sequence was analyzed by RDP Classifier (http://rdp.cme.msu.edu/) against the SILVA 16S rRNA database (Release123) using the confidence threshold of 0.7. The analysis was performed using the free online platform, Majorbio I-sanger Cloud Platform (https:// www.i-sanger.com).

\section{Statistical analysis}

The statistical analysis of alpha and beta diversity of ruminal bacterial community composition was carried out in Mothur (version v.1.30.1) (Schloss et al. 2011). The influence of sodium nitrate on growth performance, rumen fermentation parameters, microbial population and composition were analyzed using a one-way ANOVA procedure in SPSS 16.0. Data were reported as leastsquared means and standard error of means (SEM). Differences between treatments were tested using Tukey's multiple comparison tests. Differences were considered significant at $P<0.05$. Correlation analysis among the milk yield, milk composition, $\mathrm{pH}, \mathrm{VFA}, \mathrm{NH}_{3}-\mathrm{N}, \mathrm{MCP}$ concentration and microbial proportions was performed using Spearman's rank correlation, and significant differences were declared at $P<0.05$.

\section{Results}

DMI, blood methaemoglobin, milk yield and milk composition

As shown in Table 3, compared to the control group $(0 \mathrm{~g}$ nitrate/kg body weight), the nitrate additive treatments did not influence dry matter intake (DMI), milk yield and protein $(P>0.05)$. Methaemoglobin percentages of this experiment ranged from 10.2 to $17.8 \%$, with no significant difference among treatments $(P>0.05)$. Although the effects of nitrate on milk fat and total solid contents were not significant between control and nitrate treatments $(P>0.05)$, compared to the control group, sodium nitrate at $0.11 \mathrm{~g}$ nitrate $/ \mathrm{kg}$ body weight caused a decrease in milk fat and total solid contents and an increase on

Table 3 Effects of different inclusion rate of sodium nitrate on dry matter intake, milk yield and milk composition in water buffaloes

\begin{tabular}{|c|c|c|c|c|c|c|}
\hline \multirow[t]{2}{*}{ Item } & \multicolumn{4}{|c|}{ Sodium nitrate, $\mathrm{g} / \mathrm{kg}$ body weight } & \multirow[t]{2}{*}{ SE } & \multirow[t]{2}{*}{$P$} \\
\hline & 0 & 0.11 & 0.22 & 0.44 & & \\
\hline DMI (kg/day) & 13.66 & 13.08 & 13.06 & 14.08 & 0.24 & 0.389 \\
\hline Methaemoglobin, (MHB, \%) & 14.52 & 14.05 & 15.09 & 14.48 & 0.35 & 0.795 \\
\hline Milk yield (kg/day) & 7.10 & 7.01 & 7.44 & 8.01 & 0.48 & 0.899 \\
\hline Protein (\%) & 4.68 & 4.55 & 4.75 & 4.67 & 0.04 & 0.348 \\
\hline Fat (\%) & $8.54^{\mathrm{ab}}$ & $8.01^{\mathrm{a}}$ & $9.43^{b}$ & $8.95^{\mathrm{ab}}$ & 0.15 & 0.008 \\
\hline Total solid content (\%) & $19.94^{\mathrm{ab}}$ & $19.31^{\mathrm{a}}$ & $20.90^{b}$ & $20.31^{\mathrm{ab}}$ & 0.18 & 0.016 \\
\hline Lactose (\%) & $5.26^{\mathrm{a}}$ & $5.38^{b}$ & $5.20^{\mathrm{a}}$ & $5.24^{\mathrm{a}}$ & 0.02 & 0.016 \\
\hline
\end{tabular}

Different letters in the same row means significant differences $(P<0.05)$ 
lactose, while nitrate at 0.22 or $0.44 \mathrm{~g}$ nitrate $/ \mathrm{kg}$ body weight caused an increase on fat and total solid content.

\section{Fermentation characteristics}

The effect of different levels of sodium nitrate on ruminal fermentation parameters is shown in Table 4. Although the effects of nitrate on $\mathrm{pH}$ were not significant between control and nitrate treatments $(P>0.05)$, compared to the control group, ruminal $\mathrm{pH}$ values were decreased with nitrate supplementation. Here, the concentration of acetate, propionate, butyrate and total VFA in all sodium nitrate-adapted water buffaloes were greater than the control group $(P<0.05$, Table 4$)$. However, the ratio of acetate to propionate was not significantly changed ( $P$ $>0.05$ ). Compared to the control group, $\mathrm{NH}_{3}-\mathrm{N}$ and $\mathrm{MCP}$ concentration was not statistically affected by the addition of sodium nitrate in our study $(P>0.05)$.

\section{Milk fatty acids profile}

Table 5 shows the effects of sodium nitrate on milk fatty acids profile in water buffaloes. Although the milk fatty acids value at $0.11 \mathrm{~g}$ sodium nitrate $/ \mathrm{kg} / \mathrm{d}$ were slightly lower than other treatments, no significant differences were observed among different treatments $(P>0.05)$. There were no indications that inhibition of methanogenesis by sodium nitrate affected biohydrogenation involving CLA and vaccenic acid (Table 5).

\section{Microbial diversity, microbial population and PCoA analysis}

Results of the alpha index of microbial diversity of water buffaloes are resented in Table 6. Good's coverage values of all groups were higher than 0.99 , meaning that most of the bacterial and archaeal 16S rRNA sequences were present in the samples. For bacteria, the richness (ace and chao1) and diversity (Shannon index and Simpson) indices were not significantly different between the control group and nitrate treatments $(P>0.05)$. For archaea, compared to the control group, the richness (ace and chao1) and diversity (Shannon index) indices were increased by nitrate supplementation $(P<0.05)$. The Simpson values of archaea were also slightly decreased by sodium nitrate treatments $(P>0.05)$.

Tables 7 and 8 shows the effects of sodium nitrate on the average relative abundance of rumen microbiota (\% of total sequences) at the phylum and genus level in water buffaloes. In the archaeal community, Euryarchaeota was the only phylum identified. Compared with the control group, sodium nitrate did not affect bacterial abundance at the phylum and genus level, but the relative abundance of the methanogen genera was greatly changed. At the phylum level, the results revealed most of the bacterial sequences belonged to the phyla Bacteroidetes, Firmicutes, Proteobacteria. The percentages of Bacteroidetes in control, low, medium and high sodium nitrate treatments were $57.0 \%, 62.2 \%, 64.2 \%$ and $62.1 \%$, respectively. The percentages of Firmicutes in four treatments were $27.9 \%$, $31.3 \%, 19.4 \%, 23.1 \%$, respectively. The percentages of Proteobacteria in four treatments were $8.6 \%, 1.4 \%, 9.1 \%$, $7.6 \%$, respectively. The three phyla accounted for more than $90 \%$ of the sequences. Sodium nitrate numerically increased the Bacteroidetes phylum. However, there was no statistical difference in this bacterial phylum among the four treatments $(P>0.05)$.

To evaluate the effects of sodium nitrate on the ruminal bacterium community composition, the genera whose abundance were in the top 30 were selected. At the genus level, the results revealed most of the bacterial sequences belonged to the genus Prevotella, Christensenellaceae_R-7, Bacteroidales_BS11, Rikenellaceae_RC9, Acetobacter, Bacteroidales_RF16, Ruminococcaceae_NK4A214, Succiniclasticum, and Prevotellaceae_UCG-001. The nine genera accounted for more than $70 \%$ of the sequences. Five archaeal genera

Table 4 Effects of different level sodium nitrate on ruminal fermentation parameters

\begin{tabular}{|c|c|c|c|c|c|c|}
\hline \multirow[t]{2}{*}{ Parameter } & \multicolumn{4}{|c|}{ Sodium nitrate, $\mathrm{g} / \mathrm{kg}$ body weight } & \multirow[t]{2}{*}{ SE } & \multirow[t]{2}{*}{$P$} \\
\hline & 0 & 0.11 & 0.22 & 0.44 & & \\
\hline Ruminal pH & 6.85 & 6.66 & 6.73 & 6.63 & 0.04 & 0.186 \\
\hline Acetate, $\mathrm{mM}$ & $26.97^{\mathrm{a}}$ & $35.55^{b}$ & $32.67^{b}$ & $36.97^{b}$ & 1.06 & 0.001 \\
\hline Propionate, mM & $7.02^{\mathrm{a}}$ & $8.91^{b}$ & $8.01^{\mathrm{ab}}$ & $9.41^{b}$ & 0.29 & 0.010 \\
\hline Butyrate, mM & $3.91^{\mathrm{a}}$ & $5.76^{b}$ & $4.57^{\mathrm{ab}}$ & $5.29^{b}$ & 0.20 & 0.003 \\
\hline $\mathrm{A} / \mathrm{P}$ & 3.93 & 3.99 & 4.09 & 3.98 & 0.06 & 0.871 \\
\hline Total VFA, mM & $37.91^{a}$ & $50.22^{b}$ & $45.24^{\mathrm{ab}}$ & $51.67^{b}$ & 1.49 & 0.001 \\
\hline $\mathrm{NH}_{3}-\mathrm{N} \mathrm{mM}$ & $22.1^{\mathrm{ab}}$ & $27.1^{b}$ & $13.8^{\mathrm{a}}$ & $24.3^{\mathrm{ab}}$ & 1.5 & 0.022 \\
\hline $\mathrm{MCP}, \mathrm{mg} / \mathrm{ml}$ & 0.23 & 0.29 & 0.21 & 0.26 & 0.01 & 0.149 \\
\hline
\end{tabular}

Different letters in the same row means significant differences $(P<0.05)$ 
Table 5 Effects of sodium nitrate on milk fatty acids profile in water buffaloes

\begin{tabular}{|c|c|c|c|c|c|c|}
\hline \multirow[t]{2}{*}{ Fatty acids, ppm } & \multicolumn{4}{|c|}{ Sodium nitrate, $\mathrm{g} / \mathrm{kg} / \mathrm{d}$} & \multirow[t]{2}{*}{ SE } & \multirow[t]{2}{*}{$P$} \\
\hline & 0 & 0.11 & 0.22 & 0.44 & & \\
\hline C8:0 & 419.82 & 344.75 & 360.24 & 492.07 & 27.84 & 0.229 \\
\hline $\mathrm{C} 10: 0$ & 936.66 & 750.84 & 851.50 & 1088.90 & 60.16 & 0.238 \\
\hline $\mathrm{C} 11: 0$ & 2.87 & 0.98 & 3.67 & 0.99 & 0.78 & 0.519 \\
\hline C12:0 & 1239.70 & 1023.70 & 1171.50 & 1426.90 & 71.30 & 0.250 \\
\hline C13:0 & 41.36 & 32.06 & 42.15 & 40.24 & 1.95 & 0.236 \\
\hline C14:0 & $4874.8^{\mathrm{ab}}$ & $4563.6^{\mathrm{a}}$ & $4907.30^{\mathrm{ab}}$ & $6132.0^{b}$ & 213.01 & 0.045 \\
\hline C14:1 n5 & 394.40 & 394.14 & 388.84 & 452.03 & 22.11 & 0.718 \\
\hline C15:0 & 497.07 & 419.49 & 530.41 & 520.95 & 20.79 & 0.223 \\
\hline C15:1 n5 & ND & ND & ND & ND & ND & ND \\
\hline C16:0 & 20,676 & 18,459 & 21,551 & 25,071 & 879.19 & 0.059 \\
\hline C16:1 n7 & 833.28 & 818.57 & 845.94 & 981.38 & 48.23 & 0.616 \\
\hline C17:0 & 390.61 & 347.52 & 410.51 & 426.66 & 15.96 & 0.331 \\
\hline C17.1 n7 & 87.10 & 86.42 & 84.02 & 95.35 & 5.37 & 0.893 \\
\hline C18:0 & 7406.1 & 6919.6 & 7617.1 & 8292.7 & 216.07 & 0.156 \\
\hline C18:1n9c & 8256.2 & 7653.4 & $10,715.00$ & $10,195.00$ & 563.64 & 0.160 \\
\hline C18:1n9t & 1389.3 & 1244.4 & 1714.2 & 1448.9 & 63.74 & 0.066 \\
\hline C18:2n6c & 909.09 & 837.82 & 1134.2 & 1016.9 & 58.49 & 0.302 \\
\hline C18:2n6t & ND & ND & $\mathrm{ND}$ & $\mathrm{ND}$ & ND & ND \\
\hline C18:1 t11 & 1837.8 & 1074.4 & 1691.4 & 1522.6 & 188.17 & 0.515 \\
\hline C18:2 t9t11 & 1733.7 & 1706.5 & 1790.5 & 1858.8 & 129.01 & 0.978 \\
\hline C18:2 C9c11 & 656.12 & 538.28 & 765.56 & 802.16 & 49.19 & 0.224 \\
\hline C18:2 c9t11 & 391.41 & 342.23 & 450.63 & 382.53 & 30.95 & 0.673 \\
\hline $\mathrm{C} 18: 2 \mathrm{t} 10 \mathrm{c} 12$ & 20.34 & 0 & 0 & 0 & 5.08 & 0.397 \\
\hline C18:3n3 & ND & ND & ND & ND & ND & ND \\
\hline C18.3n6 & ND & ND & ND & $\mathrm{ND}$ & ND & ND \\
\hline C20:0 & 137.78 & 117.79 & 146.20 & 148.01 & 4.85 & 0.105 \\
\hline C20:1 & 46.22 & 47.67 & 59.23 & 54.12 & 3.10 & 0.423 \\
\hline C20:2 & ND & $N D$ & ND & $N D$ & ND & ND \\
\hline$C 20: 3 n 3$ & 40.23 & 34.31 & 49.43 & 42.97 & 2.46 & 0.180 \\
\hline$C 20: 3 n 6$ & ND & ND & ND & ND & ND & ND \\
\hline C20:4n6 & 58.67 & 50.16 & 71.42 & 75.84 & 4.43 & 0.151 \\
\hline$C 20: 5 n 3$ & 0.28 & 1.92 & 2.08 & 2.09 & 0.58 & 0.638 \\
\hline C21:0 & 11.03 & 6.14 & 8.76 & 8.74 & 1.30 & 0.632 \\
\hline C22:0 & 39.27 & 33.17 & 44.33 & 41.35 & 1.66 & 0.108 \\
\hline C22:1n9 & 99.65 & 126.91 & 118.62 & 80.10 & 15.99 & 0.740 \\
\hline C22:2 & ND & ND & ND & ND & ND & ND \\
\hline C22.6n3 & ND & ND & ND & ND & ND & ND \\
\hline C23:0 & 10.73 & 5.67 & 12.30 & 10.33 & 1.70 & 0.555 \\
\hline C24:0 & 23.59 & 19.98 & 30.16 & 26.53 & 1.46 & 0.089 \\
\hline$C 24: 1$ & ND & ND & ND & ND & $\mathrm{ND}$ & ND \\
\hline SFA & $36,707.41$ & $33,043.24$ & $37,685.61$ & $43,727.34$ & 1447.90 & 0.068 \\
\hline MUFA & $12,954.92$ & $11,452.11$ & $15,625.86$ & $14,837.88$ & 726.39 & 0.170 \\
\hline PUFA & 3809.81 & 3511.23 & 4263.75 & 4584.92 & 228.12 & 0.357 \\
\hline UFA/SFA & 0.4667 & 0.4584 & 0.5136 & 0.4361 & 0.0130 & 0.191 \\
\hline
\end{tabular}

Different letters in the same row means significant differences $(P<0.05)$

Sum of SFA reported in this table. Sum of MUFA reported in this table. Sum of PUFA reported in this table. Ratio between the sum of UFA and SFA 
Table 6 Effects of sodium nitrate on alpha index of microbial diversity in water buffaloes

\begin{tabular}{|c|c|c|c|c|c|c|c|}
\hline & \multirow[t]{2}{*}{ Alpha index } & \multicolumn{4}{|c|}{ Sodium nitrate $\mathrm{g} / \mathrm{kg} / \mathrm{d}$} & \multirow[t]{2}{*}{ SE } & \multirow[t]{2}{*}{$\mathbf{P}$} \\
\hline & & 0 & 0.11 & 0.22 & 0.44 & & \\
\hline \multirow[t]{5}{*}{ Bacteria } & Good's coverage & 0.9916 & 0.9929 & 0.9939 & 0.9908 & 0.008 & 0.582 \\
\hline & Chao1 & 903.45 & 864.04 & 902.73 & 929.36 & 15.82 & 0.602 \\
\hline & Shannon index & 5.01 & 5.05 & 4.94 & 5.04 & 0.0625 & 0.939 \\
\hline & Simpson & 0.0231 & 0.0166 & 0.0231 & 0.0280 & 0.0031 & 0.698 \\
\hline & Ace & 894.84 & 858.48 & 894.67 & 927.81 & 15.56 & 0.166 \\
\hline \multirow[t]{5}{*}{ Archaea } & Good's coverage & 0.9989 & 0.9987 & 0.9986 & 0.9982 & 0.0001 & 0.145 \\
\hline & Chao1 & $113.03^{\mathrm{a}}$ & $244.47^{b}$ & $208.92^{b}$ & $245.08^{b}$ & 18.43 & 0.006 \\
\hline & Shannon index & $1.0045^{\mathrm{a}}$ & $2.2233^{b}$ & $2.1112^{b}$ & $1.6401^{\mathrm{ab}}$ & 0.1779 & 0.027 \\
\hline & Simpson & 0.5515 & 0.3083 & 0.3052 & 0.4880 & 0.0458 & 0.108 \\
\hline & Ace & $136.01^{\mathrm{a}}$ & $243.57^{b}$ & $208.93^{b}$ & $245.11^{b}$ & 15.79 & 0.015 \\
\hline
\end{tabular}

Different letters in the same row means significant differences $(P<0.05)$

Table 7 Effects of sodium nitrate on average relative abundance of rumen bacterial microbiota (\% of total sequences) at the phylum level in water buffaloes $(n=3)$

\begin{tabular}{|c|c|c|c|c|c|c|}
\hline & \multirow[t]{2}{*}{ Phylum } & \multicolumn{4}{|c|}{ Relative abundance (\%) } & \multirow[t]{2}{*}{$P$ value } \\
\hline & & $0 \mathrm{~g} / \mathrm{kg}$ & $0.11 \mathrm{~g} / \mathrm{kg}$ & $0.22 \mathrm{~g} / \mathrm{kg}$ & $0.44 \mathrm{~g} / \mathrm{kg}$ & \\
\hline \multirow[t]{16}{*}{ Bacteria } & Bacteroidetes & $57.040 \pm 1.264$ & $62.240 \pm 7.488$ & $64.150 \pm 12.070$ & $62.070 \pm 14.070$ & 0.625 \\
\hline & Firmicutes & $27.890 \pm 7.445$ & $31.320 \pm 6.114$ & $19.370 \pm 3.265$ & $23.080 \pm 23.08$ & 0.184 \\
\hline & Proteobacteria & $8.628 \pm 7.151$ & $1.358 \pm 1.071$ & $9.125 \pm 7.387$ & $7.592 \pm 4.690$ & 0.206 \\
\hline & Spirochaetae & $1.163 \pm 0.788$ & $1.653 \pm 0.296$ & $1.611 \pm 0.418$ & $1.326 \pm 0.537$ & 0.746 \\
\hline & Lentisphaerae & $1.325 \pm 1.453$ & $0.797 \pm 0.940$ & $2.040 \pm 0.516$ & $1.555 \pm 1.000$ & 0.445 \\
\hline & Cyanobacteria & $1.631 \pm 2.164$ & $0.502 \pm 0.429$ & $1.618 \pm 0.991$ & $1.554 \pm 1.080$ & 0.381 \\
\hline & Saccharibacteria & $1.227 \pm 0.451$ & $0.924 \pm 0.269$ & $0.976 \pm 0.803$ & $1.450 \pm 0.091$ & 0.184 \\
\hline & Tenericutes & $0.465 \pm 0.065$ & $0.637 \pm 0.221$ & $0.398 \pm 0.292$ & $0.721 \pm 0.130$ & 0.189 \\
\hline & Actinobacteria & $0.390 \pm 0.077$ & $0.173 \pm 0.114$ & $0.422 \pm 0.363$ & $0.241 \pm 0.062$ & 0.180 \\
\hline & Fibrobacteres & $0.057 \pm 0.073$ & $0.195 \pm 0.201$ & $0.040 \pm 0.055$ & $0.041 \pm 0.036$ & 0.726 \\
\hline & Elusimicrobia & $0.033 \pm 0.028$ & $0.056 \pm 0.046$ & $0.061 \pm 0.055$ & $0.141 \pm 0.092$ & 0.449 \\
\hline & Unclassified_k_norank & $0.049 \pm 0.035$ & $0.076 \pm 0.058$ & $0.077 \pm 0.059$ & $0.072 \pm 0.082$ & 0.888 \\
\hline & SHA-109 & $0.067 \pm 0.101$ & $0.041 \pm 0.049$ & $0.056 \pm 0.052$ & $0.089 \pm 0.050$ & 0.774 \\
\hline & Verrucomicrobia & $0.008 \pm 0.003$ & $0.011 \pm 0.015$ & $0.022 \pm 0.034$ & $0.029 \pm 0.017$ & 0.421 \\
\hline & Synergistetes & $0.006 \pm 0.001$ & $0.009 \pm 0.008$ & $0.027 \pm 0.016$ & $0.026 \pm 0.012$ & 0.172 \\
\hline & Chloroflexi & $0.020 \pm 0.014$ & $0.017 \pm 0.011$ & $0.010 \pm 0.009$ & $0.016 \pm 0.007$ & 0.728 \\
\hline
\end{tabular}

were identified and compared with the control group, about $16.7-30.5 \%$ of sequences could not be classified at the genus level $(P=0.055)$. Methanobrevibacter was the most abundant genus and accounted for $95.4 \%$ of the total archaeal community in the control group. There was a tendency towards Methanobrevibacter to decrease from the sodium nitrate group $(P=0.091)$.

The abundance of total bacteria, archaea, protozoa, fungi and Butyrivibrio group species associated with fatty acid biohydrogenation was quantified to assess the impact of sodium nitrate (Table 9). No significant difference in total bacteria, methanogens, fungi, $B$. proteoclasticus, B. fibrsolvens + Pseudobutyrivibrio spp., 'Atypical' Butyrivibrio and B. hungatei abundance was observed between the control group and nitrate treatments $(P>0.05)$. PCoA analysis of bacterial and archaeal population in the rumen of water buffaloes was presented in Figs. 1 and 2. Comparisons of bacterial communities by principal coordinate analysis (PCoA) based on the Bray-Curtis distance matrix 
Table 8 Effects of sodium nitrate on average relative abundance of rumen microbial microbiota (\% of total sequences) at the genus level in water buffaloes $(n=3)$

\begin{tabular}{|c|c|c|c|c|c|c|}
\hline & \multirow[t]{2}{*}{ Genus } & \multicolumn{4}{|c|}{ Relative abundance (\%) } & \multirow[t]{2}{*}{$P$ value } \\
\hline & & $0 \mathrm{~g} / \mathrm{kg}$ & $0.11 \mathrm{~g} / \mathrm{kg}$ & $0.22 \mathrm{~g} / \mathrm{kg}$ & $0.44 \mathrm{~g} / \mathrm{kg}$ & \\
\hline \multirow[t]{30}{*}{ Bacteria } & Prevotella & $27.670 \pm 13.190$ & $42.720 \pm 6.623$ & $42.650 \pm 13.930$ & $33.770 \pm 14.870$ & 0.4987 \\
\hline & Christensenellaceae_R-7 & $8.090 \pm 3.325$ & $9.894 \pm 3.728$ & $5.634 \pm 1.645$ & $8.191 \pm 7.472$ & 0.4698 \\
\hline & Bacteroidales_BS11 & $8.432 \pm 8.314$ & $2.346 \pm 1.803$ & $3.342 \pm 1.781$ & $13.110 \pm 11.580$ & 0.4721 \\
\hline & Rikenellaceae_RC9 & $8.156 \pm 4.527$ & $4.384 \pm 1.952$ & $6.318 \pm 3.029$ & $6.783 \pm 1.719$ & 0.5267 \\
\hline & Acetobacter & $6.639 \pm 6.279$ & $0.156 \pm 0.130$ & $7.121 \pm 6.220$ & $4.391 \pm 2.977$ & 0.1743 \\
\hline & Bacteroidales_RF16 & $5.251 \pm 4.276$ & $1.602 \pm 1.431$ & $4.054 \pm 2.045$ & $2.693 \pm 1.497$ & 0.4568 \\
\hline & Ruminococcaceae_NK4A214 & $3.461 \pm 2.611$ & $5.698 \pm 2.056$ & $1.791 \pm 0.522$ & $2.219 \pm 1.076$ & 0.1811 \\
\hline & Succiniclasticum & $2.958 \pm 2.922$ & $3.227 \pm 1.716$ & $1.750 \pm 1.044$ & $0.765 \pm 0.411$ & 0.2404 \\
\hline & Prevotellaceae_UCG-001 & $2.083 \pm 1.298$ & $2.374 \pm 1.754$ & $1.595 \pm 1.075$ & $0.990 \pm 0.842$ & 0.6303 \\
\hline & Ruminococcus_2 & $1.505 \pm 0.927$ & $3.530 \pm 2.704$ & $0.709 \pm 0.555$ & $1.165 \pm 0.504$ & 0.4292 \\
\hline & Bacteroidales_S24-7 & $1.989 \pm 1.564$ & $3.023 \pm 1.262$ & $0.960 \pm 0.602$ & $0.724 \pm 0.214$ & 0.1816 \\
\hline & Unclassified_f_Prevotellaceae & $0.766 \pm 0.865$ & $3.138 \pm 2.861$ & $1.776 \pm 1.289$ & $0.742 \pm 0.476$ & 0.514 \\
\hline & Butyrivibrio_2 & $2.615 \pm 1.908$ & $1.057 \pm 0.799$ & $1.291 \pm 0.353$ & $1.309 \pm 0.834$ & 0.7466 \\
\hline & Gastranaerophilales & $1.626 \pm 2.158$ & $0.498 \pm 0.4131$ & $1.612 \pm 0.982$ & $1.455 \pm 0.953$ & 0.3803 \\
\hline & Entisphaerae_RFP12 & $1.242 \pm 1.479$ & $0.681 \pm 0.854$ & $1.855 \pm 0.477$ & $1.132 \pm 0.660$ & 0.3712 \\
\hline & Treponema_2 & $1.004 \pm 0.815$ & $1.483 \pm 0.366$ & $1.365 \pm 0.528$ & $0.953 \pm 0.435$ & 0.5641 \\
\hline & Candidatus_Saccharimonas & $1.202 \pm 0.445$ & $0.901 \pm 0.267$ & $0.936 \pm 0.779$ & $1.408 \pm 0.124$ & 0.2109 \\
\hline & Prevotellaceae_UCG-003 & $0.513 \pm 0.333$ & $0.638 \pm 0.281$ & $0.878 \pm 0.421$ & $0.441 \pm 0.177$ & 0.542 \\
\hline & Ruminococcaceae_UCG-014 & $0.389 \pm 0.230$ & $0.514 \pm 0.219$ & $0.525 \pm 0.185$ & $0.756 \pm 0.277$ & 0.5482 \\
\hline & Bacteroidales_UCG-001 & $0.365 \pm 0.144$ & $0.426 \pm 0.333$ & $0.751 \pm 0.521$ & $0.537 \pm 0.143$ & 0.5553 \\
\hline & Ruminococcaceae_UCG-010 & $0.581 \pm 0.168$ & $0.445 \pm 0.193$ & $0.337 \pm 0.089$ & $0.522 \pm 0.205$ & 0.3297 \\
\hline & Saccharofermentans & $0.514 \pm 0.239$ & $0.476 \pm 0.286$ & $0.374 \pm 0.168$ & $0.407 \pm 0.197$ & 0.8864 \\
\hline & Lachnospiraceae_XPB1014 & $0.660 \pm 0.560$ & $0.249 \pm 0.136$ & $0.477 \pm 0.164$ & $0.350 \pm 0.260$ & 0.4424 \\
\hline & Lachnospiraceae_NK3A2O & $0.440 \pm 0.120$ & $0.520 \pm 0.116$ & $0.349 \pm 0.230$ & $0.388 \pm 0.267$ & 0.7265 \\
\hline & Veillonellaceae_UCG-001 & $0.332 \pm 0.133$ & $0.274 \pm 0.166$ & $0.685 \pm 0.512$ & $0.363 \pm 0.204$ & 0.7162 \\
\hline & Ruminiclostridium_5 & $0.334 \pm 0.396$ & $0.685 \pm 0.205$ & $0.231 \pm 0.209$ & $0.322 \pm 0.224$ & 0.2355 \\
\hline & [Eubacterium]_coprostanoligenesp & $0.301 \pm 0.092$ & $0.164 \pm 0.047$ & $0.511 \pm 0.479$ & $0.368 \pm 0.118$ & 0.1561 \\
\hline & Erysipelotrichaceae_UCG-004 & $0.368 \pm 0.278$ & $0.183 \pm 0.137$ & $0.270 \pm 0.085$ & $0.416 \pm 0.090$ & 0.2641 \\
\hline & Lachnospiraceae_UCG-008 & $0.509 \pm 0.270$ & $0.216 \pm 0.121$ & $0.223 \pm 0.150$ & $0.275 \pm 0.277$ & 0.5573 \\
\hline & Succinivibrionaceae_UCG-002 & $0.364 \pm 0.331$ & $0.476 \pm 0.673$ & $0.188 \pm 0.058$ & $0.185 \pm 0.087$ & 0.7958 \\
\hline \multirow[t]{7}{*}{ Archaea } & Methanobrevibacter & $95.360 \pm 4.460$ & $64.690 \pm 25.120$ & $69.680 \pm 14.650$ & $78.230 \pm 8.953$ & 0.091 \\
\hline & Unclassified no rank & $1.363 \pm 0.464$ & $30.480 \pm 24.900$ & $25.470 \pm 13.870$ & $16.720 \pm 6.179$ & 0.055 \\
\hline & Thermoplasmatales_Incertae_Sedis & $2.651 \pm 3.902$ & $3.289 \pm 3.705$ & $4.382 \pm 1.699$ & $4.198 \pm 2.479$ & 0.917 \\
\hline & Candidatus_Methanomethylophilus & $0.583 \pm 0.999$ & $1.389 \pm 0.976$ & $0.212 \pm 0.178$ & $0.673 \pm 0.651$ & 0.380 \\
\hline & Methanosphaera & $0.041 \pm 0.013$ & $0.104 \pm 0.090$ & $0.167 \pm 0.115$ & $0.098 \pm 0.090$ & 0.368 \\
\hline & Methanobacteriaceae & $0.003 \pm 0.005$ & $0.027 \pm 0.038$ & $0.078 \pm 0.132$ & $0.053 \pm 0.046$ & 0.397 \\
\hline & Unclassified Euryarchaeota & $0.001 \pm 0.003$ & $0.024 \pm 0.027$ & $0.014 \pm 0.013$ & $0.027 \pm 0.021$ & 0.247 \\
\hline
\end{tabular}

Only top 30 were presented

showed no significant separation between the samples obtained from the water buffaloes fed control diet and diets with sodium nitrate (Fig. $1, P=0.283$ ). However, comparisons of archaeal communities by PCoA analysis showed significant separation between the control group and nitrate treatments (Fig. $2, P=0.025$ ).

\section{Correlation analysis}

The correlation among $\mathrm{pH}$, VFA, milk composition, milk yield, $\mathrm{NH}_{3}-\mathrm{N}, \mathrm{MCP}$ and dominant bacterial genera were evaluated (Fig. 3). The results showed that rumen $\mathrm{pH}$ correlated positively with Pseudobutyrivibrio abundance $(\mathrm{r}=0.759, P=0.004)$. Propionate concentration 
Table 9 Effects of sodium nitrate on microbial population in water buffaloes ( $\log _{10}$ copies/g rumen contents)

\begin{tabular}{|c|c|c|c|c|c|c|}
\hline \multirow[t]{2}{*}{ Species } & \multicolumn{4}{|c|}{ Sodium nitrate, $\mathrm{g} / \mathrm{kg}$} & \multirow[t]{2}{*}{ SE } & \multirow[t]{2}{*}{$P$} \\
\hline & 0 & 0.11 & 0.22 & 0.44 & & \\
\hline Total bacteria & 13.21 & 13.60 & 12.46 & 13.31 & 0.16 & 0.108 \\
\hline Protozoa & 8.77 & 9.00 & 7.51 & 8.46 & 0.23 & 0.142 \\
\hline Methanogenic archaea & 8.50 & 9.05 & 7.76 & 8.65 & 0.19 & 0.180 \\
\hline Fungi & 7.53 & 7.40 & 7.26 & 7.58 & 0.11 & 0.817 \\
\hline B. proteoclasticus & 8.75 & 8.77 & 7.92 & 9.21 & 0.17 & 0.087 \\
\hline $\begin{array}{l}\text { B. fibrisolvens + Pseudobutyrivibrio } \\
\text { spp. }\end{array}$ & 7.64 & 7.70 & 7.05 & 8.12 & 0.20 & 0.410 \\
\hline 'Atypical' Butyrivibrio & $8.43 a b$ & $8.67 a b$ & $7.68 \mathrm{a}$ & $9.27 b$ & 0.19 & 0.033 \\
\hline B. hungatei & 8.05 & 8.33 & 7.21 & 8.40 & 0.19 & 0.154 \\
\hline
\end{tabular}

Different letters in the same row means significant differences $(P<0.05)$

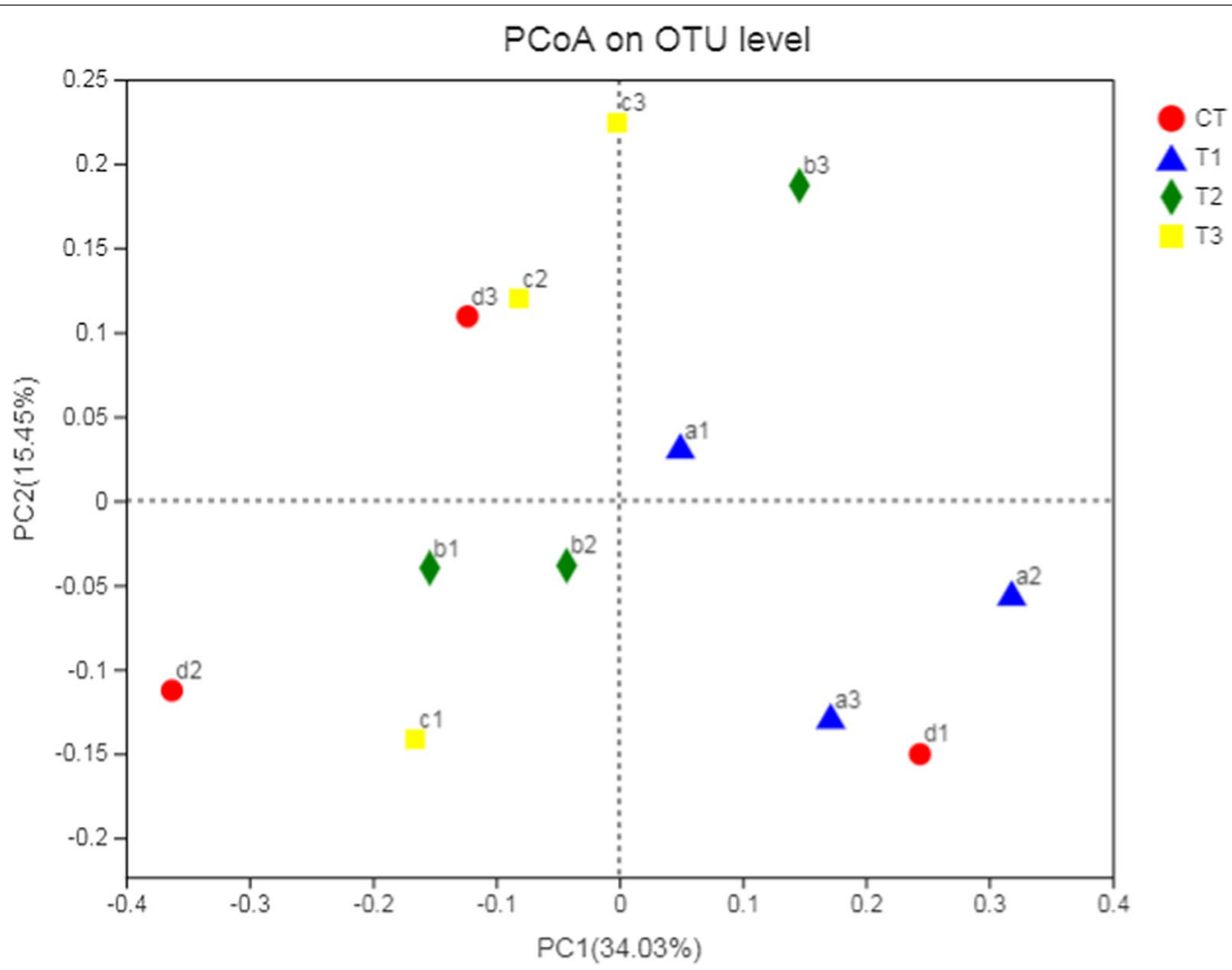

Fig. 1 PCoA analysis of bacterial population in the rumen of water buffaloes. $C T(a 1, a 2, a 3), T 1$ (b1,b2,b3), $T 2(c 1, c 2, c 3), T 3(d 1, d 2, d 3)$

correlated positively with the abundance of Victivallis ( $\mathrm{r}$ $=0.531, P=0.075)$ and Gastranaerophilales $(\mathrm{r}=0.734$, $P=0.007)$. Butyrate concentration correlated positively with Sphaerochaeta abundance $(\mathrm{r}=0.671, P=0.016)$ and negatively with the abundance of Lachnospiraceae_UCG008 ( $\mathrm{r}=-0.580, P=0.048)$ and Saccharofermentans ( $\mathrm{r}=-0.606, P=0.037)$. Acetate correlated positively with the abundance of Victivallis $(\mathrm{r}=0.636, P=0.026)$ and Sphaerochaeta $(\mathrm{r}=0.643, P=0.024)$ and negatively with Succinivibrionaceae_UCG-002 abundance ( $\mathrm{r}$ $=0.587, P=0.045)$. Total VFA concentration correlated positively with Victivallis abundance $(\mathrm{r}=0.662$, $P=0.019)$. MCP concentration positively correlation with the abundance of (Ruminococcus)_gauvreauii group ( $\mathrm{r}=0.580, P=0.048)$, Anaerovorax ( $\mathrm{r}=0.768$, $P \quad=0.004), \quad$ Ruminococcaceae_NK4A214_group (r $=0.615, P=0.033)$, Ruminiclostridium $5(\mathrm{r}=0.727, P$ $=0.007)$, Prevotellaceae_NK3B31_group $(\mathrm{r}=0.594, P$ 


\section{PCOA on OTU level}

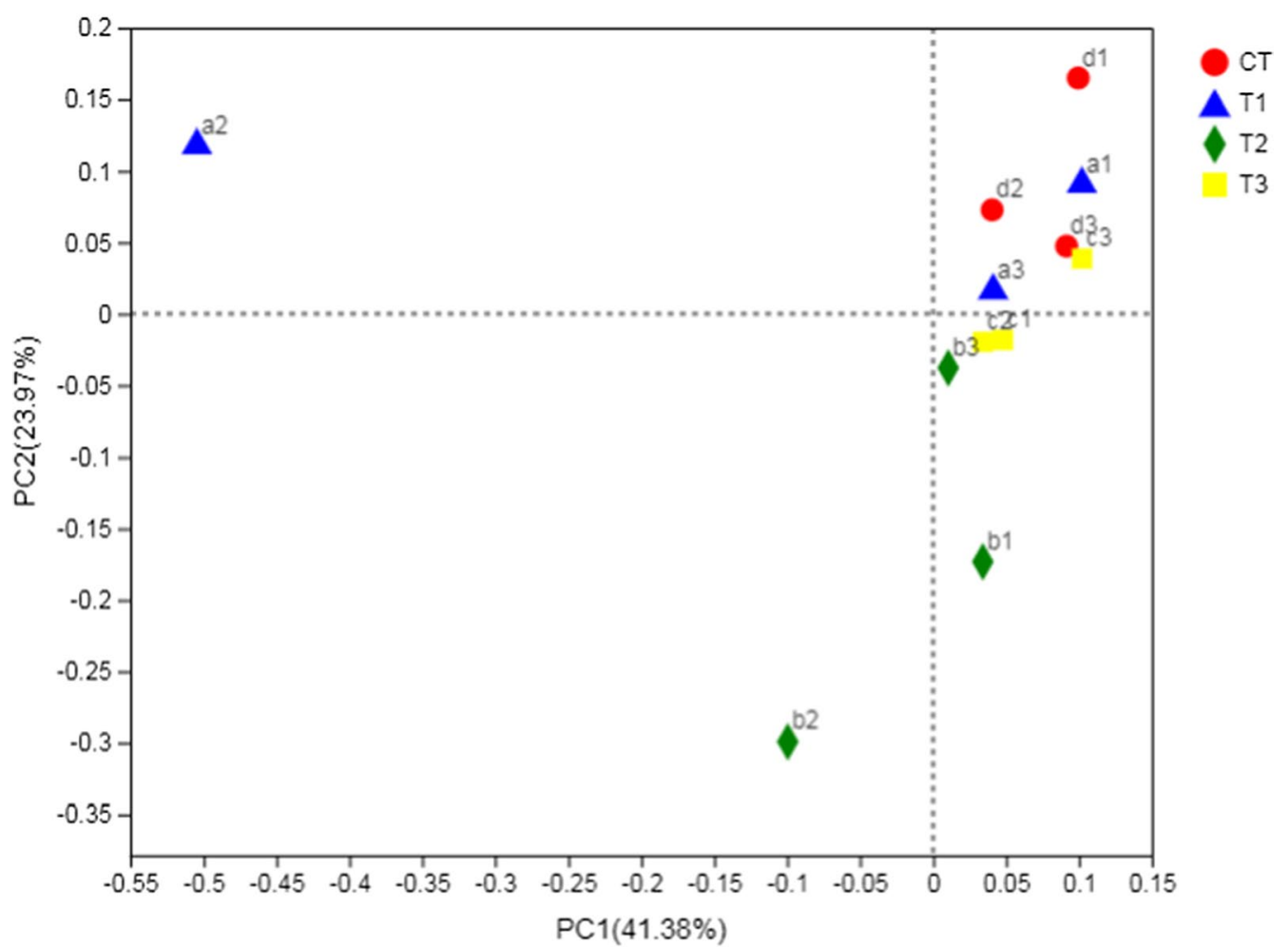

Fig. 2 Pocan analysis of archaeal population in the rumen of water buffaloes. CT (a1, a2, a3), T1 (b1, b2, b3), T2 (c1, c2, c3), T3 (d1, d2, d3)

$=0.042)$ and negatively with the abundance of Acetobacter $(\mathrm{r}=-0.683, P=0.014)$, norank_f_Clostridiales_ vadin BB60_group $(\mathrm{r}=-0.578, P=0.049)$, norank_f_Bacteroidales_RF16_group $(\mathrm{r}=-0.608, P$ $=0.036)$ and Empedobacter ( $\mathrm{r}=-0.618, P=0.032)$ genera. Milk yield correlated positively with the abundance of Lachnospiraceae UCG-008 ( $\mathrm{r}=0.734, P=0.007$ ), Ruminococcaceae UCG-005 ( $\mathrm{r}=0.581, P=0.047)$ and Lachnospiraceae_NK3A20_group $(\mathrm{r}=0.699, P=0.011)$. Milk protein correlated positively with the abundance ofnorank_o__Gastranaerophilales $(\mathrm{r}=0.655, P=0.021)$ and Veillonellaceae UCG-001 $(\mathrm{r}=0.581, P=0.048)$. The abundance of Succinivibrionaceae_UCG-002 (r $=0.622, P=0.031 ; \mathrm{r}=0.594, P=0.042)$, Prevotella $1(\mathrm{r}$ $=0.608, P=0.036 ; \mathrm{r}=0.615, P=0.033)$, Treponema $2(\mathrm{r}$ $=0.692, P=0.013 ; \mathrm{r}=0.720, P=0.008)$ and Bacteroides ( $\mathrm{r}=0.643, P=0.024 ; \mathrm{r}=0.762, P=0.004)$ displayed $\mathrm{a}$ positive correlation with milk fat and total solid contents.

The relationships between the milk yield, milk composition, $\mathrm{pH}, \mathrm{VFA}, \mathrm{NH}_{3}-\mathrm{N}, \mathrm{MCP}$ concentration and archaea proportions at the genus levels were evaluated (Fig. 4). The results showed that ruminal $\mathrm{pH}$ correlated negatively with the abundance of unclassified_k_norank ( $\mathrm{r}$ $=-0.752 ; P=0.005)$ and unclassified_p__Euryarchaeota $(\mathrm{r}=-0.759 ; P=0.004)$. Both acetate $(\mathrm{r}=-0.587 ; P$ $=0.045)$ and butyrate $(\mathrm{r}=0.583 ; P=0.046)$ were correlated positively with the abundance of unclassified p_Euryarchaeota. MCP correlated positively with the abundance of Candidatus_Methanomethylophilus ( $\mathrm{r}$ $=0.825, \quad P=0.001)$, norank_f__Thermoplasmatales Incertae_Sedis $\quad(\mathrm{r}=0.580, \quad P=0.048)$ and norank_f_Methanobacteriaceae $(\mathrm{r}=0.625, P=0.030)$. Both milk fat ( $\mathrm{r}=0.699, P=0.011)$ and total solid contents $(\mathrm{r}=0.580, P=0.047)$ were correlated positively with the abundance ofnorank_f__Thermoplasmatales_ Incertae_Sedis. The other parameters have no significant correlations with archaea genera.

\section{Discussion}

Blood methaemoglobin, DMI, milk yield and milk composition

Nitrate has been recognized as a useful and promising feed additive to reduce methane emissions from ruminants. In our present study, the nitrate concentrations of the diets and water were not determined because the basal diet and water fed to animals is the same for all treatments. Toxic levels of nitrate in feed are usually associated with excessive levels of dietary crude protein 


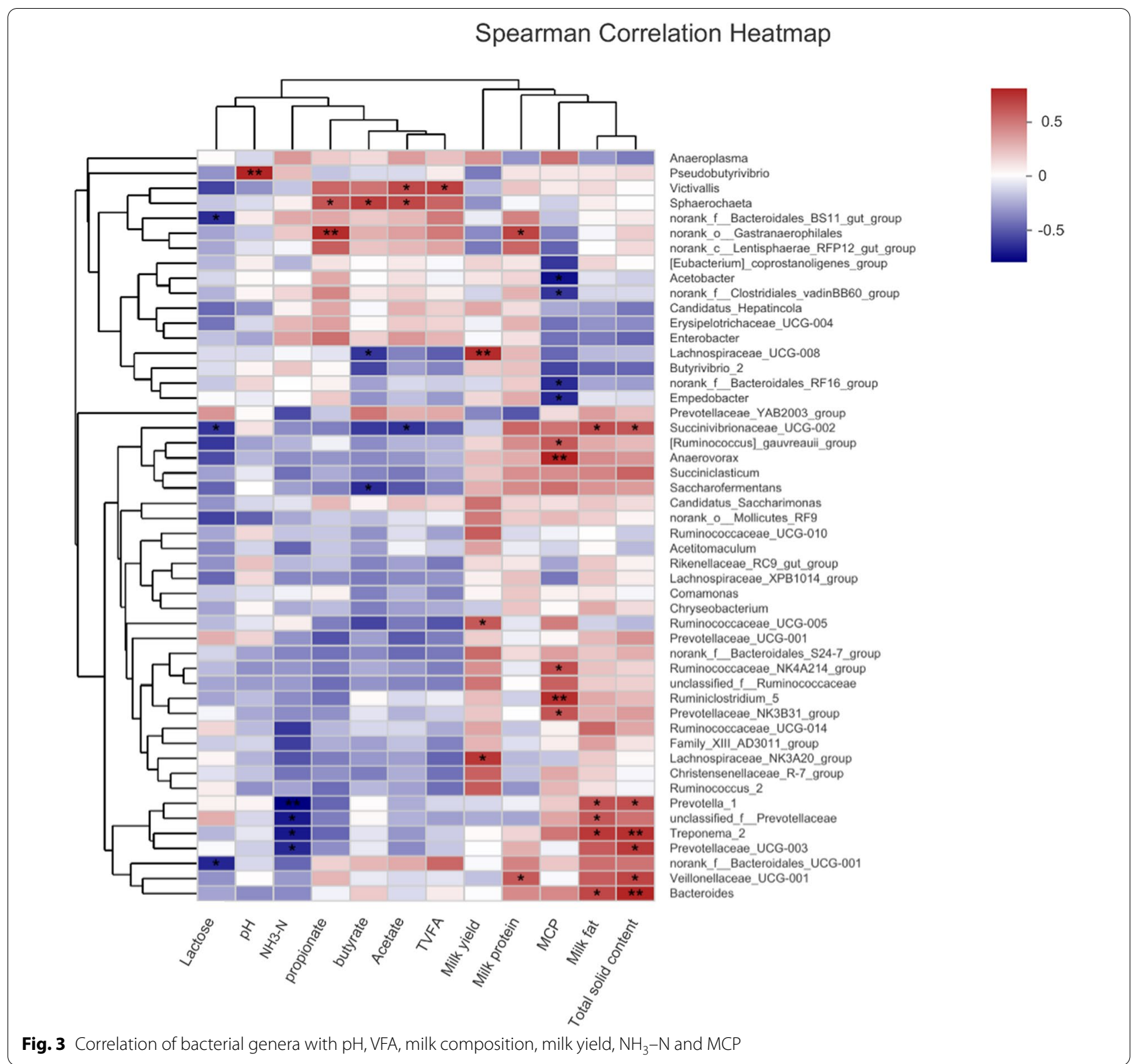

leading to high $\mathrm{NH}_{3}$ concentration in the rumen (Leng 2008). At lower inclusion levels, most ingested nitrate can convert to $\mathrm{NH}_{3}-$ Ninthe rumen, providing nitrogen for the microbial growth (Lewis 1951). Most studies have investigated the potentially toxic effects of nitrates on ruminants; however, it seems that clinical toxicity occur only when animals were given high doses of nitrate salts without a period of adaptation (Eckard 1990). The degree of intoxication can be reflected by the percentage of total haemoglobin in the blood of animals (Leng 2008). Signs of hypoxia develop when $20-30 \%$ of haemoglobin is converted to methaemoglobin. Death results when there is $70-80 \%$ of methaemoglobin (Vermunt and Visser 1987). Methaemoglobin percentages of this experiment ranged from 10.2 to $17.8 \%$, which means that the water buffaloes in this experiment were not compromised by methaemoglobin. The results are consistent with some previous in vivo studies where feeding nitrate salts did not result in clinical signs of toxicosis (Nolan et al. 2010; Van Zijderveld et al. 2011). However, other studies reported that intoxication is observed in animals when fed nitrate (Takahashi et al. 1998; Sar et al. 2004). The difference in MHB values between our study and these experiments may be caused by the means of application to the animal and nitrate dosages. In the present study, sodium nitrate was fed as a component of the diet, 


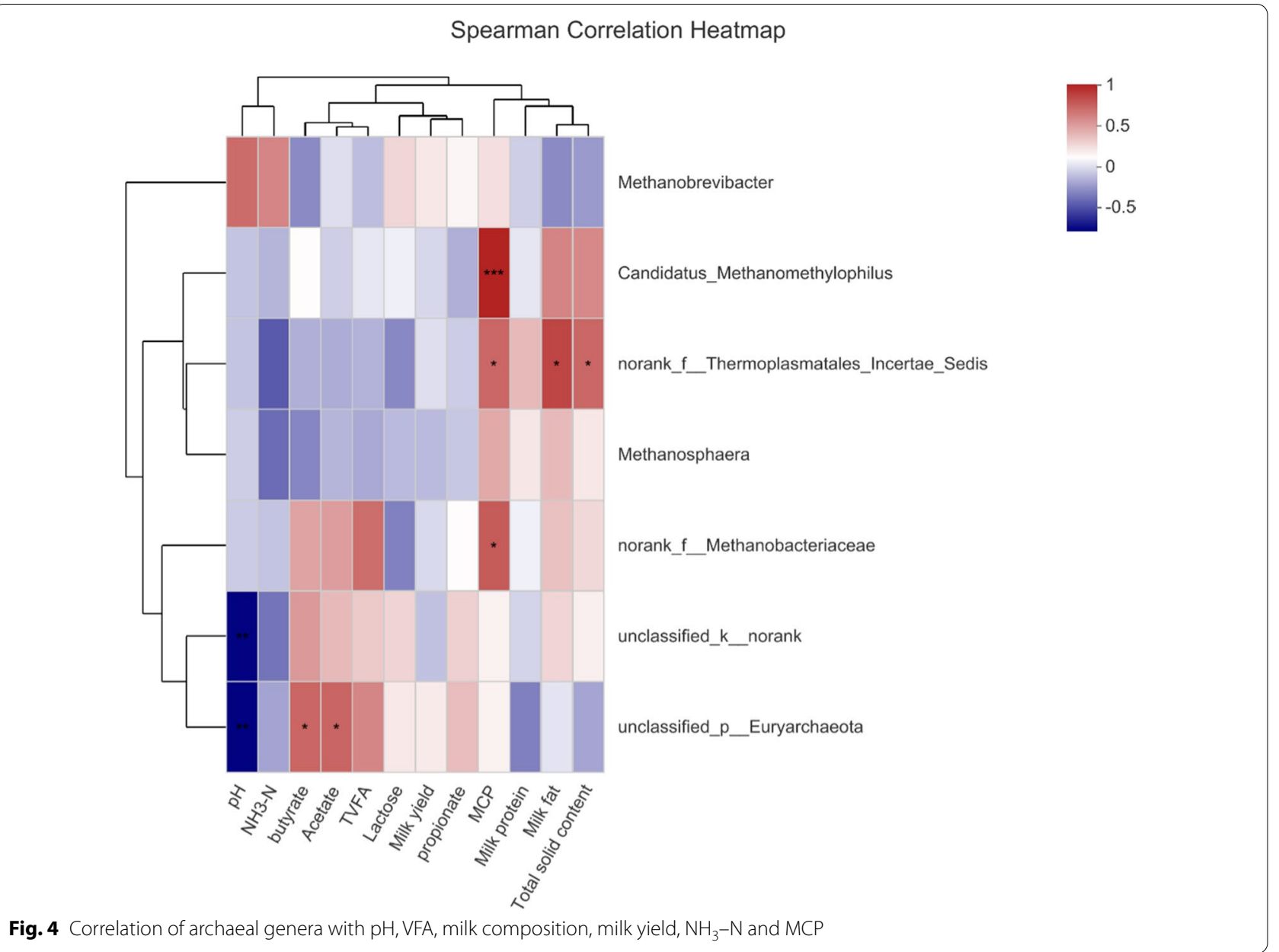

however, in the other studies, the nitrate was pulse-dosed into the rumen. Another possible reason could be the difference in animals that were not adapted to nitrate. Normally, the ruminal microbiota of adapted animals had a higher capacity to reduce nitrite than unadapted animals. Although some strategies (encapsulated nitrate, probiotics (Sar et al. 2005a) can be used to reduce the toxicity of nitrite when nitrate is used to methanogenesis inhibition, more in vivo studies for water buffaloes, including rates of metabolism of nitrate and nitrite, are still needed to understand the necessity for an acclimation strategy in these animals.

The benefits of methane reduction of ruminants (e.g., increasing propionate, butyrate and metabolizable energy supply) have been confirmed (Wolin 1960). When the blood methaemoglobin is higher than $20-30 \%$ by feeding nitrate, the ruminant's productivity will be decreased (Bruning-Fann and Kaneene1993). However, some studies reported that no adverse effects on feed intake and production when nitrate supplementation is at lower levels (Lee and Beauchemin 2014). Here, the nitrate additive treatments did not influence DMI, milk performance or milk composition except that lactose concentrations of milk were higher than control at $0.11 \mathrm{~g}$ sodium nitrate/ kg body weight (Table 3 ). Our results agree with previous studies in cattle (Newbold et al. 2014; Olijhoek et al. 2016). In contrast, other studies observed lower DMI when nitrate was fed to animals (Hulshof et al. 2012; Lee et al. 2015; Klop et al. 2016). This difference in the effects of nitrate on DMI may be contributed by the difference in diet compositions, animal species, and feeding strategy. Van Zijderveld et al. (2011) reported that fed nitrate at $2.1 \%$ of dietary DM had no adverse effects on milk production and milk composition of dairy cows. The nitrate level should be less than $2.5 \%$ of dietary DM, otherwise, the feed intake, as well as animal production, could be affected because of excessive rumen degradable protein from the non-protein nitrogen in the diet (Lee and Beauchemin 2014). Lichtenwalner et al. (1973) found that potassium nitrate of 1.0 or $2.0 \%$ in corn gluten meal- and soybean meal-based diets did not affect the growth performance of adapted finishing beef steers. There is no 
adverse effect on milk production when adequate time was allowed for cows' adaptation to the nitrate diet, even dietary inclusion of up to $5 \%$ of average daily feed intake (Farra and Satter 1971).

\section{Fermentation characteristics}

In the present study, ruminal $\mathrm{pH}$ values were decreased with nitrate supplementation, although the changes were minor and not likely to have significant effects on microbial growth or metabolism. This result was in agreement with the previous report that nitrate treatment may lead to a lower ruminal $\mathrm{pH}$ (Sar et al. 2004).

In contrast, some studies found that nitrate caused an increase in ruminal $\mathrm{pH}$ when using orchard grass forage as the substrate in vitro (Takahashi et al. 1989) or sheep (Nolan et al. 2010). Both type and level of carbohydrate supplementation can contribute to the dynamics of rumen $\mathrm{pH}$ (Wanapat et al. 2021).

Nitrate can convert to nitrite and then inhibit ruminal fermentation if ruminants were not adapted (Guo et al. 2009), while this inhibition can be removed after the animal adapted to dietary nitrate (Zhou et al. 2012). In our present study, the concentration of acetate, propionate, butyrate and total VFA in all sodium nitrateadapted water buffaloes were greater than the control group. This is consistent with what has been found in a previous study that feeding $25 \mathrm{~g}$ nitrate/ $\mathrm{kg}$ DM caused a greater total VFA concentration of sheep compared with sheep receiving an isonitrogenous amount of urea (Nolan et al. 2010). Nitrate did not consistently affect total VFA concentration, but it did shift the VFA profile to higher acetate, lower propionate and lower butyrate molar proportions. Rumen total VFA concentration was not affected by dietary nitrate treatment in beef cattle, while the proportion of acetic acid and acetate/propionate ratio tended to be greater for the nitrate diet (Hulshof et al. 2012). The increase of acetate concentration and the decrease of propionate and butyrate concentration by nitrate supplementation has been confirmed in many previous reports (Farra and Satter 1971; Nakamura 1975; Takahashi et al. 1989; Sar et al. 2004). In contrast, the in vitro study of Zhou et al. (2011), sodium nitrate greatly decreased the production of acetate and propionate. Farra and Satter (1971) also reported that fed a diet containing 20 g nitrate $/ \mathrm{kg}$ DM decreased concentrations of total VFA in dairy cows. Ruminal VFA concentration is related negatively to ruminal $\mathrm{pH}$, although the relationship appears to be weak because of large variation between diets in removal, buffering and neutralization of acids in the rumen that affects the relationship between pH and VFA (Dijkstra et al. 2012).

Some research reported that an increased ruminal $\mathrm{NH}_{3}-\mathrm{N}$ concentration is associated with nitrate supplementation (Lewis 1951). Nitrate addition can increase ammonia concentrations through respiratory nitrate ammonification in the rumen (Sar et al. 2005a, 2005b) and high ammonia concentrations can inhibit methanogens (Chen et al. 2007, 2008). Increasing ruminal $\mathrm{NH}_{3}-\mathrm{N}$ could provide a ruminal nitrogen source leading in increased microflora and increased microbial protein synthesis and rumen fiber digestion (Khejornsart et al. 2011). In contrast, a difference in $\mathrm{NH}_{3}-\mathrm{N}$ was not expected in the present study. This was consistent with Nolan et al. (2010) which reported no difference in ruminal $\mathrm{NH}_{3}-\mathrm{N}$ between the sheep fed urea or nitrate. Factors which may explain this difference are the different roughage source and type of protein in the diet (true protein and non-protein nitrogen), supply of fermentable energy and efficiency of microbial protein synthesis (Wanapat et al. 2014). MCP concentration was also not statistically affected by the addition of sodium nitrate in our study, which is consistent with the report of Lund et al. (2014). Ruminal MCP synthesis depends mainly on an adequate supply of carbohydrates as an energy source for the synthesis of peptide bonds (Bach et al. 2005).

\section{Milk fatty acids profile}

The effect of feeding nitrate on milk fatty acids profile is little known in water buffaloes or other ruminant species, however, it might be anticipated that the diversion of reducing equivalents away from methanogenesis could have consequences for other processes involving oxidation/reduction reactions such as propionogenesis and fatty acid biohydrogenation. To our knowledge, only one study reported the effect of nitrate on milk fatty acids composition in vivo (Klop et al. 2016). Klop et al. reported that nitrate had no effect on SFA proportion and proportion of MUFA, but increased the proportion of C4:0, C14:0 iso, C15:0 iso, C15:0 anteiso, C16:0 trans-9, C17:0, C18:0, C18:1 trans-10, C18:1 trans-11, C18:2 cis-9, trans-11, C18:3n-6, C20:0 and PUFA in milk fatty acids (Klop et al. 2016). CLA and vaccenic acid (trans-11-18:1) are intermediates in the biohydrogenation of linoleic acid to the fully saturated stearic acid (Jenkins et al. 2008). In the present experiment, there were no indications that inhibition of methanogenesis by sodium nitrate affected biohydrogenation involving CLA and vaccenic acid. Our previous in vitro study also suggested that inhibiting methanogenesis have no unintended deleterious consequences on fatty acid metabolism in the rumen (Yang et al. 2019). The reason is probably only about $1-2 \%$ of hydrogen was consumed by biohydrogenation as compared to methanogenesis (Nagaraja et al. 1997). 


\section{Microbial abundance changes}

Bacteroidetes is a major non-cellulosic plant constituent degrader, also an important proteolytic phylum, in the rumen (Thoetkiattikul et al. 2013). Prevotella is an important genus of Bacteroidetes phylum. In the present study, we observed that the relative abundance of the top genus of Prevotella were increased by sodium nitrate. This result is consistent with the previous study (Patra and Yu 2013; Zhao et al. 2015). Low nitrate addition can increase the relative abundance of non-cellulose degraders while high-level nitrate inhibited them (Zhao et al. 2015). In the present study, feeding nitrate caused Chao1, Shannon index and ace values of archaea to be higher than the control group. It indicates the diversion of $\mathrm{H}_{2}$ towards nitrate reduction had a significant effect on the archaeal community. The relative abundance of the Methanobrevibacter genus in sodium nitrate treatments tended to be lower than the control group whereas the relative abundance of an archaeal genus (unclassified no rank) tended to be increased. In contrast, Zhao et al. (2018) found the relative abundance of the majority of the genera in this study, vadinCA11 and Methanobrevibacter, was not significantly influenced by nitrate. Methanosphaera and Methanimicrococcus abundance increased linearly commensurate with increasing nitrate, while Methanoplanus abundance was significantly decreased.

B. proteoclasticus, B. fibrsolvens + Pseudobutyrivibrio spp., 'Atypical' Butyrivibrio and B. hungatei are members of Butyrivibrio group and carry out the biohydrogenation of unsaturated fatty acids and the formation of CLA and VA (Paillard et al. 2007; Lourenço et al. 2010). In the present study, the population of total bacteria, fungi, methanogens and Butyrivibrio group members did not differ from the control group. Our results may indicate that adaptation enabled rumen microorganisms to maintain their abundance in the rumen community. These results were consistent with previous studies. Patra and $\mathrm{Yu}$ (2014) similarly investigated the effects of nitrate $(5 \mathrm{mM})$ on the rumen microbial community and abundances of select microbial populations using in vitro methods and found that nitrate treatment did not alter the abundances of total bacteria, Ruminococcus albus, or archaea. Zijderveld et al. (2010) reported that the protozoal population in the rumen of lambs was unaffected by the inclusion of nitrate in the diet. Nitrate supplementation did not affect 16S rRNA copies of bacteria, protozoa, methanogens, or fungi (Wang et al. 2018). Lin et al. (2011) found that the nitrate-reducing activity of a fungal fraction from ruminal digesta was low, so their contribution to nitrate metabolism is likely to be minor. In contrast, the populations of representative cellulolytic bacteria, $F$. succinogenes, $R$. flavefaciens and $R$. albus, methanogens, protozoa and fungi were decreased by feeding $9 \mathrm{~g} /$ day of potassium nitrate (Asanuma et al. 2015). Compared with the control, archaeal populations were considerably decreased in the nitrate-inoculated media, however, no differences in total bacterial populations (Zhou et al. 2011, 2012). The number of methanogens decreased when nitrate was included in the diet, however, the protozoa population was unaffected (Zijderveld et al. 2010). Many of these differences are likely due to the difference in nitrate dose and perhaps to the different techniques employed for nitrate inclusion. Even after adaptation to dietary nitrate, the relative population sizes for all three putative nitrate-reducing species were very low (Lin et al. 2013). These results indicate that some bacteria can adapt to nitrate or its reduction intermediates, while others probably cannot.

\section{Association of rumen microbial abundance with fermentation parameters}

Correlation analysis indicated a cluster of bacteria positively correlated with $\mathrm{pH}$ and VFA, including Pseudobutyrivibrio, Victivallis, Gastranaerophilales, Sphaerochaeta, signifying their importance in VFA synthesis. Members of the genus Pseudobutyrivibrio are commonly found in the rumen and reported to use a wide range of soluble and some insoluble substrates and characteristically ferment carbohydrates to butyrate, formate, lactate, and acetate (Moon et al. 2008; Kopečnýet al. 2003). Victivallis can use fructose as its only energy and carbon source and produces acetate, ethanol, $\mathrm{H}_{2}$, and bicarbonate as fermentation products from glucose (Janssen and Hedlund 2011). Sphaerochaeta are known to utilize various sugars and to produce volatile fatty acids as fermentation end products. Gastranaerophilales belongs to class Melainabacteria, which is capable of fermenting arrange of sugars (e.g., glucose, starch and hemicellulose) into butyrate in the gut of herbivores (Rienzi et al. 2013). Prevotella is a dominant genus of Bacteroidetes phylum and displayed positive correlations with milk fat and total solid contents and negatively with $\mathrm{NH}_{3}-\mathrm{N}$ in this study. Prevotella exhibited a lesser importance in methane emissions because it associated with nitrogen metabolism and the pentose phosphate pathway (Hassanet al. 2021; Martínez-Álvaro et al. 2020). In this study, ruminal $\mathrm{pH}$ correlated negatively with the abundance of unclassified Euryarchaeota. This is agreed with previous study (Diaz et al. 2017). We observed a lower Euryarchaeota: Bacteria ratio and abundance of Methanobrevibacter sp. in the rumen of sodium nitrate supplementation, supported that sodium nitrate is a feed additive that affects enteric methane emissions persistently. This result is consistent with previous study (Granja-Salcedo et al. 2019). The lack of significant correlations between some microbial abundance with VFA does not suggest that those 
microbial taxa are unimportant. More work needed to explore the correlations, because a small number of species might have a strong impact on rumen fermentation parameters. In conclusion, added 0.11-0.44 g sodium nitrate/kg of body weight increased VFA production and the archaeal richness and diversity indices in water buffaloes but had no effect on milk yield, fatty acids profile, bacterial or archaeal abundance and Butyrivibrio group population related to biohydrogenation. Thus, unintended deleterious consequences of lowering methane emissions using dietary nitrate in water buffaloes are unlikely.

\section{Acknowledgements}

We would like to thank Professor Robert John Wallace for his valuable comments during the preparation of this manuscript.

\section{Authors' contributions}

$\mathrm{CY}$ designed the overall study and wrote the manuscript, $\mathrm{FX}, \mathrm{ZT}, \mathrm{XL}, \mathrm{CW}, \mathrm{YG}$ and KP performed the experiments. All the authors contributed to the article and approved the submitted version. All authors read and approved the final manuscript.

\section{Funding}

This study was supported by the National Natural Science Foundation of China (N0.31560649), National Modern Agricultural Industry Technology System Guangxi Dairy Buffalo Innovation Team Project (nycytxgxcxtd-2021-21) and the Guangxi Natural Science Foundation (2018GXNSFAA281162).

\section{Availability of data and materials}

Data and materials will be made available on reasonable request. The raw sequences were deposited in the NCBI Sequence Read Archive (SRA) database (Bacterial Accession Number: SRR11450759; Archaeal Accession Number: SRR11476468)

\section{Declarations}

\section{Ethics approval and consent to participate}

All applicable institutional guidelines for the care and use of animals were followed.

\section{Consent for publication}

Not applicable.

\section{Competing interests}

The authors declare no competing interests.

Received: 11 October 2021 Accepted: 22 January 2022

Published online: 05 February 2022

\section{References}

Ahmad S, Zhang T, Lee F, Liu Y, Li X, Guo M (2013) Seasonal variations in chemical composition of buffalo milk. Buffalo Bull 32(Special Issue 2):1324-1329 Asanuma N, Yokoyama S, Hino T (2015) Effects of nitrate addition to a diet on fermentation and microbial populations in the rumen of goats, with special reference to Selenomonas ruminantium having the ability to reduce nitrate and nitrite. Anim Sci J86(4):378-384

Bach A, Calsamiglia S, Stern MD (2005) Nitrogen metabolism in the rumen. J Dairy Sci 88:E9-E21

Bruning-Fann C, Kaneene J (1993) The effects of nitrate, nitrite, and N-nitroso compounds on animal health. Vet Hum Toxicol 35(3):237-253

Buddle BM, Denis M, Attwood GT, Altermann E, Janssen PH, Ronimus RS, Pinares-Patiño CS, Muetzel S, Wedlock DN (2011) Strategies to reduce methane emissions from farmed ruminants grazing on pasture. Vet J 188(1):11-17

Chen J, Yu Z, Michel FC, Wittum T, Morrison M (2007) Development and application of real-time PCR assays for quantification of erm genes conferring resistance to macrolides-lincosamides-streptogramin B in livestock manure and manure management systems. Appl Environ Microb 73(14):4407-4416

Chen Y, Cheng JJ, Creamer KS (2008) Inhibition of anaerobic digestion process: a review. Bioresour Technol 99(10):4044-4064

Denman SE, McSweeney CS (2006) Development of a real-time PCR assay for monitoring anaerobic fungal and cellulolytic bacterial populations within the rumen. FEMS Microb Ecol 58(3):572-582

Denman SE, Tomkins NW, McSweeney CS (2007) Quantitation and diversity analysis of ruminal methanogenic populations in response to the antimethanogenic compound bromochloromethane. FEMS Microb Ecol 62(3):313-322

Di Rienzi SC, Sharon I, Wrighton KC, Koren O, Hug LA, Thomas BC, Goodrich JK, Bell JT, Spector TD, Banfield JF, Ley RE (2013) The human gut and groundwater harbor non-photosynthetic bacteria belonging to a new candidate phylum sibling to Cyanobacteria. Elife 2(2):e01102. https://doi. org/10.7554/eLife.01102

Diaz CJM, Cabral C, Redondo LM, Pin ND, Colombatto D, Farber MD, Fernandez MME (2017) Impact of chestnut and quebracho tannins on rumen microbiota of bovines. Biomed Res Int. https://doi.org/10.1155/2017/9610810

Dijkstra J, Ellis JL, Kebreab E, Strathe AB, Lopez S, France J, Bannink A (2012) Ruminal $\mathrm{pH}$ regulation and nutritional consequences of low $\mathrm{pH}$. Anim Feed Sci Tech 172(1-2):22-33

Eckard R (1990) The relationship between the nitrogen and nitrate content and nitrate toxicity potential of Lolium multiflorum. J Grassland Soc South Afr 7(3):174-178

FAO (2020) https://www.fao.org/faostat/en/\#data/OCL/visualize

Farra P, Satter L (1971) Manipulation of the ruminal fermentation. III. Effect of nitrate on ruminal volatile fatty acid production and milk composition. Dairy Sci 54(7):1018-1024

Gerber PJ, Steinfeld H, Henderson B, Mottet A, Opio C, Dijkman J, Falcucci A, Tempio G (2013) Tackling climate change through livestock: a global assessment of emissions and mitigation opportunities. Food and Agriculture Organization of the United Nations (FAO), Rome

Givens DI, Shingfield K (2004) Foods derived from animals: the impact of animal nutrition on their nutritive value and ability to sustain long-term health. Nutr Bull 29(4):325-332

Granja-Salcedo YT, Fernandes RM, Araujo RCD, Kishi LT, Berchielli TT, Resende FDD, Berndt A, Siqueira GR (2019) Long-term encapsulated nitrate supplementation modulates rumen microbial diversity and rumen fermentation to reduce methane emission in grazing steers. Front Microbiol 10:614

Guo W, Schaefer D, Guo X, Ren L, Meng Q (2009) Use of nitrate-nitrogen as a sole dietary nitrogen source to inhibit ruminal methanogenesis and to improve microbial nitrogen synthesis in vitro. Asian Aust J Anim Sci 22(4):542-549

Guyader J, Eugène M, Meunier B, Doreau M, Morgavi D, Silberberg M, Rochette Y, Gerard C, Loncke C, Martin C (2015) Additive methane-mitigating effect between linseed oil and nitrate fed to cattle. J Anim Sci 93(7):3564-3577

Hassan FU, Guo Y, Li M, Tang Z, Peng L, Liang X, Yang C (2021) Effect of methionine supplementation on rumen microbiota, fermentation, and amino acid metabolism in in vitro cultures containing nitrate. Microorganisms 9(8):1717

Hulshof RBA, Berndt A, Gerrits WJJ, Dijkstra J, Van Zijderveld SM, Newbold JR, Perdok HB (2012) Dietary nitrate supplementation reduces methane emission in beef cattle fed sugarcane-based diets. J Anim Sci 90(7):2317-2323

Janssen PH, Hedlund BP (2011) Family I. Victivallaceae fam. Nov. In: Krieg NR, Staley JT, Hedlund BP, Paster BJ, Ward N, Ludwig W, Whitman WB (eds) Bergey's manual of systematic bacteriology, vol 4. Springer, New York, pp 795-799

Jenkins T, Wallace R, Moate P, Mosley E (2008) Board-invited review: recent advances in biohydrogenation of unsaturated fatty acids within the rumen microbial ecosystem. J Anim Sci 86(2):397-412

Khejornsart P, Wanapat M, Rowlinson P (2011) Diversity of anaerobic fungi and rumen fermentation characteristic in swamp buffalo and beef cattle fed on different diets. Livest Sci 139(3):230-236 
Klop G, Hatew B, Bannink A, Dijkstra J (2016) Feeding nitrate and docosahexaenoic acid affects enteric methane production and milk fatty acid composition in lactating dairy cows. J Dairy Sci 99(2):1161-1172

Kopečný J, Zorec M, Mrazek J, Kobayashi Y, Marinšek-Logar R (2003) Butyrivibrio hungatei sp. nov. and Pseudobutyrivibrio xylanivorans sp. nov. butyrate-producing bacteria from the rumen. Int I Syst Evol Microbiol 53(1):201-209

Lee C, Beauchemin KA (2014) A review of feeding supplementary nitrate to ruminant animals: nitrate toxicity, methane emissions, and production performance. Can J Anim Sci 94(4):557-570

Lee C, Araujo R, Koenig K, Beauchemin K (2015) Effects of encapsulated nitrate on enteric methane production and nitrogen and energy utilization in beef heifers. J Anim Sci 93(5):2391-2404

Leng RA (2008) The potential of feeding nitrate to reduce enteric methane production in ruminants. A report to the department of climate change. University of New England, Canberra

Lewis $D$ (1951) The metabolism of nitrate and nitrite in the sheep. 1. The reduction of nitrate in the rumen of the sheep. Biochem J 48(2):175-180

Lichtenwalner R, Fontenot J, Tucker R (1973) Effect of source of supplemental nitrogen and level of nitrate on feedlot performance and vitamin $A$ metabolism of fattening beef calves. J Anim Sci 37(3):837-847

Lin M, Schaefer D, Guo W, Ren L, Meng Q (2011) Comparisons of in vitro nitrate reduction, methanogenesis, and fermentation acid profile among rumen bacterial, protozoal and fungal fractions. Asian Aust J Anim Sci 24(4):471-478

Lin M, Guo W, Meng Q, Stevenson DM, Weimer PJ, Schaefer DM (2013) Changes in rumen bacterial community composition in steers in response to dietary nitrate. Appl Microb Biot 97(19):8719-8727

Lourenço M, Ramos-Morales E, Wallace R (2010) The role of microbes in rumen lipolysis and biohydrogenation and their manipulation. Animal 4(7):1008-1023

Lund P, Dahl R, Yang H, Hellwing ALF, Cao B, Weisbjerg MR (2014) The acute effect of addition of nitrate on in vitro and in vivo methane emission in dairy cows. Anim Prod Sci 54(9):1432-1435

Makkar H, Sharma O, Dawra R, Negi S (1982) Simple determination of microbial protein in rumen liquor. J Dairy Sci 65(11):2170-2173

Martínez-Álvaro M, Auffret MD, Stewart RD, Dewhurst RJ, Duthie CA, Rooke JA, Wallace RJ, Shih B, Freeman TC, Watson M, Roehe R (2020) Identification of complex rumen microbiome interaction within diverse functional niches as mechanisms affecting the variation of methane emissions in bovine. Front Microbiol 11:659

Moon CD, Pacheco DM, Kelly WJ, Leahy SC, Li D, Kopečný J, Attwood GT (2008) Reclassification of Clostridium proteoclasticum as Butyrivibrio proteoclasticus comb. nov., a butyrate-producing ruminal bacterium. Int J Syst Evol Microbiol 58(9):2041-2045

Nagaraja TG, Newbold CJ, Van Nevel CJ, Demeyer DI (1997) Manipulation of ruminal fermentation. In: Hobson PN, Stewart CS (eds) The rumen microbial ecosystem. Springer, Dordrecht, pp 523-632

Nakamura Y (1975) Nitrate metabolism of microorganisms in the rumen of sheep fed high nitrate forages. Jpn J Zootech Sci 47:63-67

Newbold JR, Van Zijderveld SM, Hulshof RBA, Fokkink WB, Leng RA, Terencio P, Powers WJ, Van Adrichem PSJ, Paton ND, Perdok HB (2014) The effect of incremental levels of dietary nitrate on methane emissions in Holstein steers and performance in Nelore bulls. J Anim Sci 92(11):5032-5040

Nolan JV, Hegarty R, Hegarty J, Godwin I, Woodgate R (2010) Effects of dietary nitrate on fermentation, methane production and digesta kinetics in sheep. Anim Prod Sci 50(8):801-806

Olijhoek D, Hellwing ALF, Brask M, Weisbjerg MR, Højberg O, Larsen MK, Dijkstra J, Erlandsen EJ, Lund P (2016) Effect of dietary nitrate level on enteric methane production, hydrogen emission, rumen fermentation, and nutrient digestibility in dairy cows. J Dairy Sci 99(8):6191-6205

Pachauri RK, Allen MR, Barros VR, Broome J, Cramer W, Christ R, Church JA, Clarke L, Dahe Q, Dasgupta P, Dubash NK, Edenhofer O, Elgizouli I, Field CB, Forster P, Friedlingstein P, Fuglestvedt J, Gomez-Echeverri L, Hallegatte S, Hegerl G, Howden M, Jiang K, Jimenez CB, Kattsov V, Lee H, Mach KJ, Marotzke J, Mastrandrea MD, Meyer L, Minx J, Mulugetta Y, O'Brien K, Oppenheimer M, Pereira JJ, Pichs-Madruga R, Plattner GK, Pörtner HO, Power SB, Preston B, Ravindranath NH, Reisinger A, Riahi K, Rusticucci M, Scholes R, Seyboth K, Sokona Y, Stavins R, Stocker TF, Tschakert P, Van VD, Van YJP (2014) Climate change 2014: synthesis report. Contribution of
Working Groups I, II and III to the fifth assessment report of the Intergovernmental Panel on Climate Change. IPCC, Geneva

Paillard D, McKain N, Chaudhary LC, Walker ND, Pizette F, Koppova I, McEwan NR, Kopečný J, VercoePE LP, Wallace RJ (2007) Relation between phylogenetic position, lipid metabolism and butyrate production by different Butyrivibrio-like bacteria from the rumen. Antonie Van Leeuwenhoek 91(4):417-422

Patra AK, Yu Z (2013) Effective reduction of enteric methane production by a combination of nitrate and saponin without adverse effect on feed degradability, fermentation, or bacterial and archaeal communities of the rumen. Bioresour Technol 148:352-360

Patra AK, Yu Z (2014) Combinations of nitrate, saponin, and sulfate additively reduce methane production by rumen cultures in vitro while not adversely affecting feed digestion, fermentation or microbial communities. Bioresour Technol 155:129-135

Qin W (1982) Determination of rumen volatile fatty acids by means of gas chromatography. J Nanjing Agric Coll 4:110-116

Sar C, Santoso B, Mwenya B, Gamo Y, Kobayashi T, Morikawa R, Kimura K, Mizukoshi H, Takahashi J (2004) Manipulation of rumen methanogenesis by the combination of nitrate with $\beta 1-4$ galacto-oligosaccharides or nisin in sheep. Anim Feed Sci Tech 115(1-2):129-142

Sar C, Mwenya B, Pen B, Takaura K, Morikawa R, Tsujimoto A, Kuwaki K, Isogai N, Shinzato I, Asakura Y, Toride Y, Takahashi J (2005a) Effect of ruminal administration of Escherichia coli wild type or a genetically modified strain with enhanced high nitrite reductase activity on methane emission and nitrate toxicity in nitrate-infused sheep. Brit J Nutr 94(5):691-697

Sar C, Mwenya B, Santoso B, Takaura K, Morikawa R, Isogai N, Asakura Y, Toride Y, Takahashi J (2005) Effect of Escherichia coli wild type or its derivative with high nitrite reductase activity on in vitro ruminal methanogenesis and nitrate/nitrite reduction. J Anim Sci 83(3):644-652

Schloss PD, Gevers D, Westcott SL (2011) Reducing the effects of PCR amplification and sequencing artifacts on $16 \mathrm{~S}$ rRNA-based studies. PloS ONE 6(12):e27310

Shingfield KJ, Kairenius P, Ärölä A, Paillard D, Muetzel S, Ahvenjärvi S, Vanhatalo A, Toivonen V, Huhtanen P, Griinari JM, Wallace RJ (2012) Dietary fish oil supplements modify ruminal biohydrogenation, alter the flow of fatty acids at the omasum, and induce changes in the ruminal Butyrivibrio population in lactating cows. J Nutr 142(8):1437-1448

Sylvester JT, Karnati SK, Yu Z, Morrison M, Firkins JL (2004) Development of an assay to quantify rumen ciliate protozoal biomass in cows using real-time PCR. J Nutr 134(12):3378-3384

Takahashi J, Johchi N, Fujita H (1989) Inhibitory effects of sulphur compounds, copper and tungsten on nitrate reduction by mixed rumen micro-organisms. Brit J Nutr 61(3):741-748

Takahashi J, Ikeda M, Matsuoka S, Fujita H (1998) Prophylactic effect of L-cysteine to acute and subclinical nitrate toxicity in sheep. Anim Feed Sci Tech 74(3):273-280

Takai K, Horikoshi K (2000) Rapid detection and quantification of members of the archaeal community by quantitative PCR using fluorogenic probes. Appl Environ Microb 66(11):5066-5072

Thoetkiattikul H, Mhuantong W, Laothanachareon T, Tangphatsornruang S, Pattarajinda V, Eurwilaichitr L, Champreda V (2013) Comparative analysis of microbial profiles in cow rumen fed with different dietary fiber by tagged 16S rRNA gene pyrosequencing. Curr Microb 67(2):130-137

Van Zijderveld S, Gerrits W, Apajalahti J, Newbold J, Dijkstra J, Leng RA, Perdok HB (2010) Nitrate and sulfate: effective alternative hydrogen sinks for mitigation of ruminal methane production in sheep. J Dairy Sci 93(12):5856-5866

Van Zijderveld S, Gerrits W, Dijkstra J, Newbold J, Hulshof R, Perdok H (2011) Persistency of methane mitigation by dietary nitrate supplementation in dairy cows. J Dairy Sci 94(8):4028-4038

Vermunt J, Visser R (1987) Nitrate toxicity in cattle. N Z Vet J 35(8):136-137

Wanapat M, Gunun P, Anantasook N, Kang S (2014) Changes of rumen pH, fermentation and microbial population as influenced by different ratios of roughage (rice straw) to concentrate in dairy steers. J Agri Sci 152(4):675-685

Wanapat M, Viennasa B, Matra M, Totakul P, Phesatcha B, Ampapon T, Wanapat $S$ (2021) Supplementation of fruit peel pellet containing phytonutrients to manipulate rumen $\mathrm{pH}$, fermentation efficiency, nutrient digestibility and microbial protein synthesis. J Sci Food Agric. https://doi.org/10.1002/ jsfa.11096 
Wang R, Wang M, Ungerfeld EM, Zhang XM, Long DL, Mao HX, Deng JP, Bannink A, Tang ZL (2018) Nitrate improves ammonia incorporation into rumen microbial protein in lactating dairy cows fed a low-protein diet. J Dairy Sci 101(11):9789-9799

Weatherburn M (1967) Phenol-hypochlorite reaction for determination of ammonia. Anal Chem 39(8):971-974

Wolin MJ (1960) A theoretical rumen fermentation balance. J Dairy Sci 43(10):1452-1459

Yang C, Rooke JA, Cabeza I, Wallace RJ (2016) Nitrate and inhibition of ruminal methanogenesis: microbial ecology, obstacles, and opportunities for lowering methane emissions from ruminant livestock. Front Microb 7:132

Yang C, McKain N, McCartney CA, Wallace RJ (2019) Consequences of inhibiting methanogenesis on the biohydrogenation of fatty acids in bovine ruminal digesta. Anim Feed Sci Tech 254:114189

Yu Z, Morrison M (2004) Improved extraction of PCR-quality community DNA from digesta and fecal samples. Biotechniques 36(5):808-812

Yu Z, Michel FC, Hansen G, Wittum T, Morrison M (2005) Development and application of real-time PCR assays for quantification of genes encoding tetracycline resistance. Appl Environ Microb 71(11):6926-6933

Zhang L (2007) Feed analysis and feed quality testing technology, 3rd edn. Chinese agricultural Press, Beijing

Zhao L, Meng O, Ren L, Liu W, Zhang X, Huo Y, Zhou Z (2015) Effects of nitrate addition on rumen fermentation, bacterial biodiversity and abundance. Asian Aust J Anim Sci 28(10):1433

Zhao L, Meng Q, Li Y, Wu H, Huo Y, Zhang X, Zhou Z (2018) Nitrate decreases ruminal methane production with slight changes to ruminal methanogen composition of nitrate-adapted steers. BMC Microb 18(1):1-8

Zhou Z, Meng Q, Yu Z (2011) Effects of methanogenic inhibitors on methane production and abundances of methanogens and cellulolytic bacteria in vitro ruminal cultures. Appl Environ Microb 77(8):2634-2639

Zhou Z, Yu Z, Meng Q (2012) Effects of nitrate on methane production, fermentation, and microbial populations in vitro ruminal cultures. Bioresour Technol 103(1):173-179

\section{Publisher's Note}

Springer Nature remains neutral with regard to jurisdictional claims in published maps and institutional affiliations.

\section{Submit your manuscript to a SpringerOpen ${ }^{\circ}$ journal and benefit from:}

- Convenient online submission

- Rigorous peer review

- Open access: articles freely available online

- High visibility within the field

- Retaining the copyright to your article

Submit your next manuscript at $\boldsymbol{\nabla}$ springeropen.com 